Cahiers « Mondes anciens »

ANCIENS

Histoire et anthropologie des mondes anciens

4 | 2013

Journées doctorales ANHIMA 2010 et 2011

\title{
Sistemi di immagini, sistemi di oggetti
}

Le loutrophoroi del pittore di Baltimora

Monica Baggio

\section{(2) OpenEdition}

Journals

Edizione digitale

URL: http://journals.openedition.org/mondesanciens/1072

DOI: 10.4000/mondesanciens. 1072

ISSN: 2107-0199

Editore

UMR 8210 Anthropologie et Histoire des Mondes Antiques

Notizia bibliografica digitale

Monica Baggio, « Sistemi di immagini, sistemi di oggetti », Cahiers " Mondes anciens » [En ligne],

4 | 2013, mis en ligne le 01 juillet 2013, consulté le 20 avril 2019. URL : http://journals.openedition.org/ mondesanciens/1072; DOI : 10.4000/mondesanciens.1072

Questo documento è stato generato automaticamente il 20 aprile 2019

\section{(c) (†) $\ominus$}

Les Cahiers «Mondes Anciens » sont mis à disposition selon les termes de la licence Creative Commons Attribution - Pas d'Utilisation Commerciale - Pas de Modification 4.0 International. 


\title{
Sistemi di immagini, sistemi di oggetti
}

\author{
Le loutrophoroi del pittore di Baltimora
}

Monica Baggio

1 Il repertorio figurativo offerto dalla produzione apula della seconda metà del IV secolo a.C. si rivela un osservatorio privilegiato per indagare, attraverso la combinazione dei segni messi in sistema nelle immagini, le forme di auto-rappresentazione del genere femminile ${ }^{1}$. In particolare, in questa sede, focalizzeremo la nostra attenzione sulle potenzialità semantiche e comunicative che determinati oggetti, tradizionalmente legati al mondo femminile, veicolano nel momento in cui dalla pratica quotidiana passano al mondo delle immagini dipinte ${ }^{2}$, dove superano la mera funzione pratica per qualificarsi come segni portatori di valori simbolici e rappresentativi dello status dei personaggi rappresentati ${ }^{3}$.

2 Che gli oggetti giochino un ruolo importante nelle società antiche appare chiaro già in un magistrale saggio di Louis GERNET (1948), apparso nel Journal de Psychologie col titolo La notion mythique de la valeur en Grèce ${ }^{4}$, che costituisce la prima messa a punto teorica di un discorso sugli oggetti nella cultura greca. Secondo lo studioso vi sono dei racconti mitici in cui gli oggetti, preziosi agalmata o keimelia, occupano un ruolo centrale, il cui statuto si carica di complesse valenze simboliche in grado di conferire a chi li possiede un grande prestigio.

3 Su questa linea si muovono numerosi lavori di François LISSARRAGUE (1995) e Françoise FRONTISI-DUCROUX (1998), volti ad indagare il complesso tema dell'identità nei vasi attici, che hanno dato corretta lettura dei significati simbolici di determinati oggetti legati al mondo femminile (come scatole, contenitori, specchi ${ }^{5}$, etc.) dimostrando come essi « had some relevance, not only because they are connected with female activities but also because symbolic values connected with these activities defined, in the eyes of the painters and their clients, the status of women $»^{6}$, veicolando cioè statuti, ruoli e identità. In relazione alla produzione attica scrive ancora F. LISSARRAGUE $(2006$, p. 15) : « les objets retenus par les peintres sont en nombre limité, liés à des champs d'activités précis, et porteurs de valeurs sémantiques fortes ». 
Per quanto riguarda il mondo magnogreco, dove il ricorso alle possibilità di significazione delle immagini si rivela fondamentale in un contesto per molti versi sfuggente, in quanto privo di una tradizione scritta che possa aiutarci a delineare i diversi aspetti dell'identità femminile, già da tempo tutto un filone di studi insiste sull'importante ruolo giocato dagli oggetti nell'immaginario figurato: degli anni'70 sono due contributi di H.R.W. SMITH, l'uno dal titolo Deadlocks? (1970) e l'altro dal titolo Funerary simbolisme in Apulian vase painting (1976), nei quali lo studioso metteva bene in evidenza tutta la complessità del linguaggio iconografico apulo, caratterizzato da una costante sovrapposizione di temi e livelli di lettura ${ }^{7}$. Più o meno negli stessi anni Jean-Marc MORET (1978), in un importante contributo dal titolo Jugement de Pâris en Grande-Grèce: mythe et actualité politique, dedica un intero paragrafo alla polisemia del linguaggio iconografico apulo dal titolo Dénotation et Connotation. Le "symbolisme» funéraire apulien (MORET 1978, p. 85) : il merito dello studioso è quello di porre al centro dell'attenzione il sistema di convenzioni che regola la rappresentazione visuale, di cui gli oggetti costituiscono una parte integrante. Egli dimostra come, per comprendere pienamente il significato di questi, si debba ricorrere ad un doppio piano di lettura: al piano denotativo, che veicola il significato primario o convenzionale dell'oggetto si accompagna sempre il piano connotativo, che rimanda ad un secondo livello di significazione, più concettuale, che accresce il contenuto semantico di ciascun oggetto, caricandolo di molteplici sfumature di significato. Ne sono un esempio le osservazioni che lo studioso conduce sul ruolo di determinati oggetti, quali la conchiglia (MORET 1978, p. 89 e nota 85) oppure l'hydria (MORET 1978, p. 90), il cui significato viene di volta in volta analizzato a seconda del contesto in cui si trovano inseriti. Merito dello studioso è anche quello di portare l'attenzione sul ruolo giocato dall'osservatore, che non è mai figura passiva nei confronti del mondo delle immagini ma che, attraverso l'esercizio mnemonico, richiama, perché riconosce, tutta la rete di funzioni e significati connessi ai differenti oggetti ${ }^{8}$. Questa posizione viene ripresa in seguito da Christian AELLEN (1994, p. 15) e, più di recente, da Thomas MORARD (2009), quest'ultimo in un volume assai interessante dedicato alla genesi e al significato delle assemblee divine nella ceramica apula della seconda metà del IV secolo a.C. Qui lo studioso dedica un intero paragrafo a Le langage des objets, analizzando in particolare il ruolo che determinati oggetti, quali il sacco da viaggio o l'hydria giocano nell'immagine, dimostrando come esso sia ambivalente e veicoli ora valori concreti, ora valori simbolici. In più occasioni anche Hélène Cassimatis ha indagato le valenze simboliche di singoli oggetti, secondo una prospettiva tutta interna alla produzione magnogreca, che insiste sulla specificità dell'iconografia italiota $\mathrm{e}$ sulle sue differenti categorie di rappresentazione rispetto alla ceramica attica. Analizzando in particolare le occorrenze ed il significato di oggetti quali il kalathos ( CASSIMATIS 1990), gli oggetti della cosmetica (CASSIMATIS 1993), in particolare gli specchi ( CASSIMATIS 1999) ${ }^{9}$, la studiosa ritiene che questi prendano rilevanza non solo in relazione ai singoli personaggi ma, addirittura, in rapporto alle singole scene, sottolineando come sia proprio il sistema degli oggetti ad orientare il significato delle scene più che i singoli personaggi ${ }^{10}$.

5 Sulla scorta di questa breve premessa, nella prospettiva antropologica di delineare, attraverso la selezione degli oggetti messi in sistema nelle immagini, qualche riflessione sulle forme di autorappresentazione femminile in seno alla comunità apula, interessanti considerazioni sembrano emergere dall'analisi della struttura dell'immagine di un piccolo gruppo di vasi, in particolare loutrophoroi prodotte nel corso degli ultimi trent'anni del IV secolo a.C. nella bottega del Pittore di Baltimora, uno dei protagonisti della fase detta 
tardo apula ${ }^{11}$. Un attento riesame di tutta la produzione di questo pittore, la cui conoscenza è aumentata nel corso degli ultimi trent'anni, lo pone quale erede della bottega del pittore di Dario e degli Inferi ${ }^{12}$ e localizza la sua produzione nell'area nordapula, tra i centri di Ruvo, Canosa ed $\mathrm{Arpi}^{13}$. Il dato si rivela di estremo interesse, perché pone l'attività del pittore in connessione con un momento di cambiamento sia a livello di officine ceramiche, che da Taranto si sarebbero spostate verso l'Apulia settentrionale ${ }^{14}$, sia in relazione al tipo di committenza, da ricercare nella locale aristocrazia indigena oramai ellenizzata ${ }^{15}$ che, a partire dalla metà del IV secolo a.C., avrebbe utilizzato le produzioni vascolari come veicolo di affermazione e di legittimazione ${ }^{16}$. In particolare, nella vasta produzione del pittore di Baltimora ${ }^{17}$, concentreremo la nostra attenzione su un piccolo corpus costituito da otto loutrophoroi del tipo "barrel amphorae", tutte a destinazione funeraria ${ }^{18}$. Come ben noto, in ambito apulo le loutrophoroi, vaso di norma destinato già in ambiente greco ${ }^{19}$ a contenere l'acqua, cominciano a comparire dopo la metà del IV secolo a.C. prevalentemente nei corredi femminili : assai prestigiosi per dimensioni, per la qualità elevata delle caratteristiche tecniche e per il livello di pittura, questi contenitori sono per tradizione legati alla vita della donna, in particolare alla purificazione, al bagno rituale della sposa e, per lo stretto legame tra cerimonie nuziali e rituali funerari, anche alla toletta della defunta ${ }^{20}$ : è possibile dunque dovessero essere esibiti come oggetti d'apparato durante le cerimonie funebri per poi accompagnarla nella sepoltura. La scelta del campione, per quanto piuttosto ristretto, è tuttavia giustificata dalla forte coerenza che i documenti offrono sul piano delle soluzioni iconografiche adottate, dal fatto che escono dalla stessa bottega, hanno la stessa forma e, a dispetto della loro totale decontestualizzazione, sono riferibili al consumo di una circostanziata area geografica.

L'analisi iconografica consentirà, come vedremo, un doppio ordine di considerazioni : sul piano dell'analisi formale ed iconografica, in quanto la presenza degli oggetti determina le modalità di costruzione dello spazio da rappresentare; sul piano dell'analisi antropologica, in quanto permette una riflessione sul sistema di oggetti usati per costruire l'identità di specifici personaggi rappresentati. 
Fig. 1 : Loutrophoros apula a figure rosse

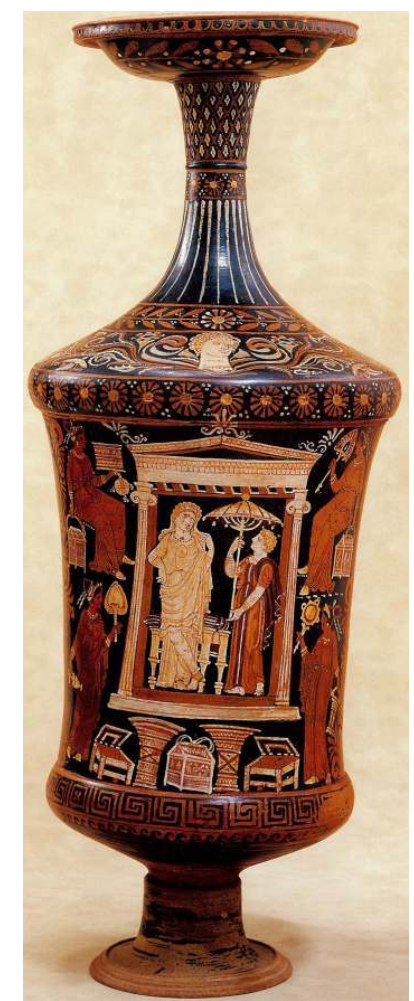

Avignone, Museo Calvet, inv. 23504

cassimatis 2000 , fig. 23A

7 Come punto di partenza possiamo prendere la loutrophoros ora conservata al museo di Avignone (23504), proveniente dal mercato antiquario (Fig. 1) ${ }^{21}$ : il pittore di Baltimora organizza la scena intorno al naiskos, segno grafico che polarizza l'attenzione di tutte le componenti dell'immagine ${ }^{22}$, connotandola come funeraria e veicolando, al contempo, un' immediata forma di eroizzazione della defunta rappresentata ${ }^{23}$.

8 All'interno dell'edicola, dipinta di bianco e dotata di una certa profondità, come si evince dalla resa prospettica del soffitto a cassettoni, sono dipinte due figure femminili: quella di sinistra, di proporzioni maggiori, con vesti molto eleganti e gioielli, simbolo di rango elevato $^{24}$, tiene con una mano un lembo del velo sollevato nel tipico gesto dello svelamento, l'anakalypsis ${ }^{25}$, che connota la donna nella condizione di sposa. Accanto, una giovane fanciulla più piccola di statura, forse un'ancella, è colta mentre regge un parasole, a sottolineare per mezzo del gesto e dell'oggetto la posizione sociale ed il rango della defunta ${ }^{26}$. Le due donne sono completamente dipinte di bianco, come bianco è il colore del parasole che la giovane tiene con la mano per la punta inferiore ${ }^{27}$. I ritocchi di bianco sovraddipinti, che fanno parte dei modi espressivi del pittore di Baltimora, se da un lato traducono il pallore specifico dell'incarnato femminile ${ }^{28}$, tradizionale segno distintivo di bellezza, dall'altro evidentemente richiamano la realtà materica del marmo, usato nei realia per raffigurare le statue dei defunti entro naiskoi ${ }^{29}$. Un cenno d'ambientazione interna è dato dal diphros, posto in secondo piano, che suggerisce metaforicamente nell'edificio funerario il nuovo oikos della defunta ${ }^{30}$. Se, come ben è stato scritto, « avec l'iconographie du naiskos présence et figuration du défunt sont perçues de façon complémentaire selon un schéma que l'on pourrait qualifier de re-présentation ${ }^{31}$, 
anche in questo caso la defunta è rappresentata non nel mondo reale ma in quello ideale dell'Aldilà, eroizzata in virtù del suo ruolo e del suo rango. Intorno al naiskos, si dispongono quattro figure femminili di offerenti ${ }^{32}$, probabilmente « beate » che accolgono nell'Aldilà la nuova compagna, intente a celebrare per mezzo dei doni e dei gesti rituali l'immagine ideale della defunta rappresentata nella sua condizione di sposa ${ }^{33}$.

Nell'organizzazione dell'immagine l'elemento più interessante ritengo sia la strutturazione della parte inferiore del vaso, dove alcuni oggetti sono presentati in maniera ordinata, accostati e disposti stoichedon sulla linea del meandro ${ }^{34}$. Come in un fregio sono allineati, regolarmente spaziati, si distinguono per la cura minuziosa con cui sono resi e per le dimensioni, leggermente superiori ai consueti moduli : l'oggetto centrale è una cista, finemente lavorata, ai lati della quale si dispongono simmetricamente due kalathoi e due cofanetti aperti ${ }^{35}$. Come è ben noto, questi oggetti sono tradizionalmente legati al mondo femminile, strettamente uniti tra loro da precise connessioni semantiche, scelti ed associati nel campo figurato in quanto contribuiscono a definire in qualche modo l'universo funzionale della defunta ${ }^{36}$. Il cofanetto, come ha esaurientemente dimostrato F. LISSARRAGUE (1995), occupa una posizione rilevante nel mondo femminile già nella pittura vascolare attica, dove compare sia come contenitore funzionale (di gioielli, alabastra, etc.) sia come segno portatore di significati simbolici connessi con le attività femminili della kosmesis e della tesaurizzazione ${ }^{37}$; i grandi kalathoi simboleggiano il lavoro domestico di rango ${ }^{38}$, sottolineano l'identità femminile ${ }^{39}$, l'attività economica della donna nell'ambito dell'oikos familiare ${ }^{40} \mathrm{e}$, di conseguenza, la sua identità sociale $^{41}$.

Il dato importante da ritenere è che il pittore qui, in maniera poco consueta, ha scelto di raffigurare gli oggetti al di fuori del contesto tradizionale della manipolazione, dell'offerta e dello scambio. Posati a terra, monumentalizzati, non manipolati, tutti questi oggetti hanno una funzione allo stesso tempo estetizzante e semantica: essi sono ornamento della superficie vascolare ma, in quanto dotati di una propria autonomia, diventano segni, enunciati ${ }^{42}$ dello status della defunta, al pari degli oggetti deposti nelle tombe. A ragione Hélène CASSIMATIS (2000), cui si deve la prima edizione di questo vaso, lo ritiene frutto di una specifica committenza, che intende celebrare, attraverso la particolare composizione della scena, una precisa defunta in qualità di sposa e signora dell'oikos ${ }^{43}$. 
Fig. 2 : Loutrophoros apula a figure rosse

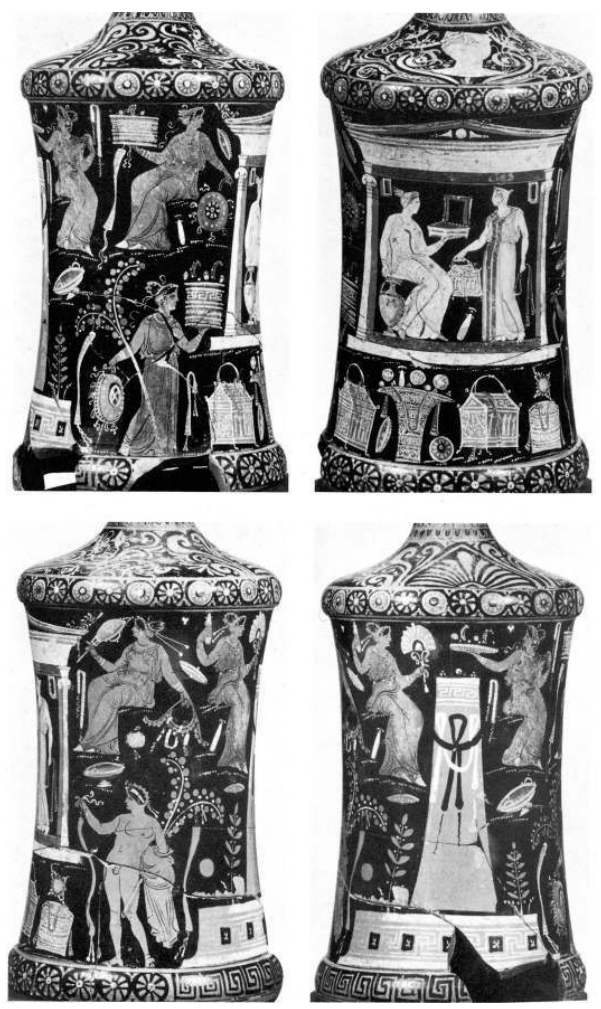

Basilea, Mercato antiquario

RVAP II, PL. 330

Un dispositivo analogo si ritrova in un'altra loutrophoros del pittore di Baltimora ${ }^{44}$, anch'essa purtroppo dal mercato antiquario (Fig. 2). Al centro della scena è ancora il naiskos con all'interno due donne colte nell'atto di scambiarsi oggetti: a sinistra una giovane è seduta su un'hydria ${ }^{45}$ e regge con una mano un cofanetto aperto ; davanti a lei, un'altra giovane fanciulla stante tiene una cista lavorata ed un nastro ; posato al suolo tra loro, totalmente defunzionalizzato, un alabastron contenitore di profumi ${ }^{46}$. Dal punto di vista figurativo possiamo osservare come il pittore abbia collocato sull'asse centrale all'interno dell'edicola il cofanetto aperto, la cista, l'alabastron per contenere profumi, tutti attributi che consentono l'accesso allo statuto matrimoniale, che compongono un sistema di segni che rinvia al mundus muliebris, all'ambito della kosmesis e, in definitiva, alla conquista della charis in una situazione evocativa di un rito di passaggio ${ }^{47}$.

Come di consueto, altri oggetti entrano in gioco nell'immagine: al di fuori del naiskos, offerenti, in pose rituali, recano offerte per il defunto (ciste, tamburi, corone, nastri, fronde vegetali, patere). Tuttavia, anche in questo caso, il tratto interessante è la profusione di oggetti che occupano lo spazio ai piedi del naiskos e che prendono un senso per dimensioni, posizione e ridondanza. Si riconoscono una cista con manico lavorata, una patera vista di profilo, che rimanda al rito, un kalathos carico di offerte (delle balle di lana ${ }^{48}$, un alabastron per profumi ed un nastro ${ }^{49}$ ), ancora una patera, questa volta vista di fronte, una cista, ancora una cista di foggia diversa associata ad un nastro e ad uno specchio ${ }^{50}$. Se, da un lato, l'abbondanza e la reduplicazione degli oggetti rappresentati intende qualificare immediatamente il prestigio economico della defunta, dall'altro, ancora una volta, gli oggetti non sono presentati come insieme funzionale: isolati, non 
manipolati ma, nello stesso tempo semanticamente legati tra loro, essi funzionano come una lista di attributi, per così dire, "grammaticali » a partire dai quali si costruisce l'identità.

13 Non c'è alcun intento narrativo nella raffigurazione di questo vaso, molto probabilmente destinato ad una giovane donna che forse non ha conosciuto pienamente le nozze (come sembrerebbe indicare la presenza della palla, oggetto la cui valenza simbolica evoca tanto i giochi infantili quanto uno stadio d'età prematrimoniale ${ }^{51}$, e della cintura sciolta, anche questo oggetto allusivo all'unione sessuale ${ }^{52}$ ) : la protome tra girali fioriti dipinta sul collo, il cui significato simbolico è ancor oggi al centro del dibattito $\mathrm{critico}^{53}$, come la sequenza ordinata e ritmata degli oggetti che si snodano nella parte inferiore del vaso, potrebbero ben evocare, insieme allo spazio del naiskos, luoghi simbolici dove proiettare in una prospettiva escatologica il rango e i valori della defunta, secondo un ordine ideale dopo il caos della morte ${ }^{54}$.

Fig. 3 : Loutrophoros apula a figure rosse

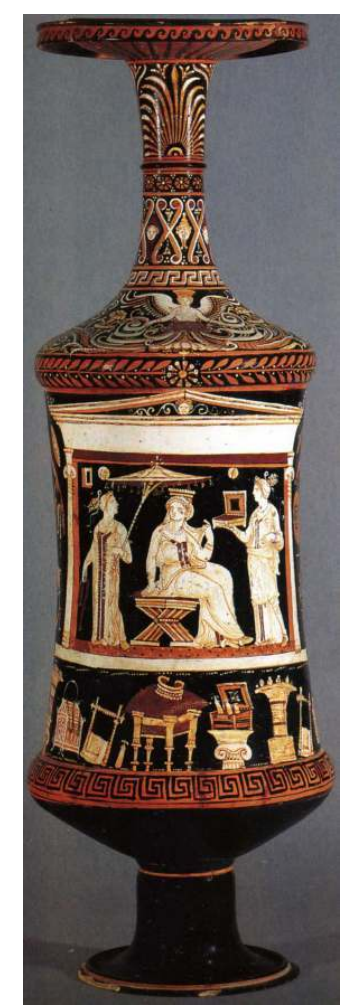

Matera, Museo Archeologico Nazionale « D. Ridola », inv. 164531

MITI gRECI 2004, P. 206, fig. 1

Su una loutrophoros conservata a Matera ${ }^{55}$ (Fig. 3) il dispositivo di celebrazione eroica della defunta, attraverso la moltiplicazione degli oggetti, è ancora più esplicito. All'interno del naiskos due figure femminili, quella a destra con un parasole mentre quella di sinistra con un cofanetto aperto ${ }^{56}$, si organizzano in rapporto ad una donna seduta su diphros al centro, colta mentre compie il gesto dell'anakalypsis, gesto che veicola precisi significati erotici e matrimoniali. In essa dobbiamo certamente riconoscere la defunta, rappresentata con molta eleganza attraverso una serie di attributi che sanciscono l'accesso allo statuto matrimoniale: il diadema sul $\mathrm{capo}^{57}$, gli orecchini ${ }^{58}$, i bracciali, le vesti sontuos $\mathrm{e}^{59}$ magnificano da un lato la sua bellezza e il potere di seduzione, dall'altro il 
prestigio economico ${ }^{60}$. A rafforzare questo concetto contribuisce ancora una volta la sequenza di oggetti allineata ai piedi dell'edicola funeraria che, accentuando l'accumulo, comunicano il prestigio sociale della defunta: da sinistra, un capitello ionico regge un kalathos caricato di uova o frutti, a sottolineare forse la sfera della fecondità e della riproduzione $e^{61}$, una cista con manico decorata, una cetra, forse richiamo alla paideia femminile ${ }^{62}$, un alabastron per contenere oli profumati, uno sgabello su cui poggia una corona dorata, un capitello che regge un cofanetto aperto contenente tre alabstra ${ }^{63}$, un cofanetto con sopra un kalathos caricato di uova o dolci, ancora una cetra e un cofanetto. Tutti questi oggetti, totalmente slegati da un preciso contesto di utilizzo, costruiscono un sistema di autorappresentazione dalla forte ed unitaria connotazione, evidentemente funzionale a restituire alla defunta la sua identità.

A sottolineare ulteriormente il valore semantico dei segni iconici selezionati, contribuiscono anche le particolari corrispondenze che si intuiscono all'interno dell'immagine: ponendo sullo stesso asse il parasole, tradizionale segno di rango elevato ${ }^{64}$, la defunta col capo coronato di diadema e, nel fregio inferiore, lo sgabello sul quale poggia un secondo diadema, il pittore sembra voler organizzare un sistema di segni che esalta la donna nell'aspetto matrimoniale, eroizzandola.

Veramente in quest'immagine tutti gli oggetti funzionano come una sorta di kosmos, una parure di cui si orna la defunta, in linea con numerosi esempi tratti dalla tradizione letteraria, in particolare da Euripide, dove il termine kosmos viene spesso usato anche in un contesto funerario per designare la parure o le offerte ai morti ${ }^{65}$.

Fig. 4 : Loutrophoros apula a figure rosse
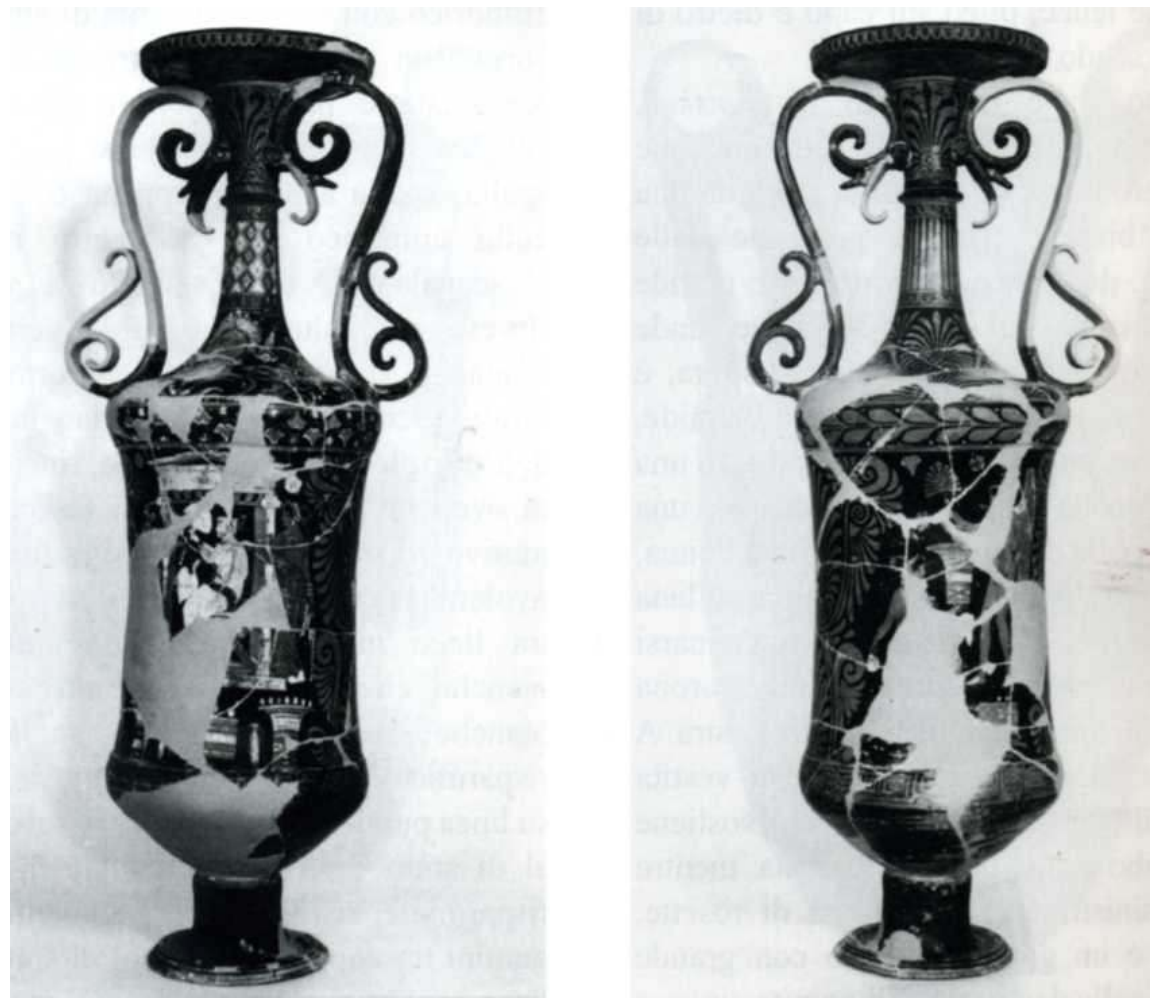

Bari, Museo Archeologico Provinciale, inv. 2397 MONTANARO 2007, P. 410, fig. 314 
17 In maniera più sintetica rispetto agli esemplari sin qui analizzati, tuttavia simile per impianto, è la loutrophoros apula, rinvenuta a Ruvo ed ora conservata a Bari (Museo Archeologico Provinciale, inv. 2397)66, attribuita al pittore della Patera (Fig. 4). Secondo Trendall e Cambitoglou ${ }^{67}$, questo pittore fu uno stretto collaboratore della bottega del pittore di Baltimora, del quale sembra riprendere schemi iconografici ${ }^{68}$. Sul corpo del vaso è raffigurato un naiskos con all'interno una donna seduta, dipinta di bianco; regge nella mano destra un ventaglio mentre, con la sinistra, sembra voler offrire qualcosa ad un cigno poggiato sulla sua coscia. Il cigno, come è noto, è animale notoriamente sacro ad Afrodite, riconducibile alla sfera matrimoniale-matronale, nel suo aspetto riproduttivo. Ai lati dell'edicola funeraria sono raffigurate due donne, delle offerenti, che recano rispettivamente una patera ed una benda. Ai piedi del monumento ritroviamo ancora una sequenza di oggetti costituita da due alabastra, due grossi kalathoi ed una cista : anche in quest'ultimo caso, come negli esempi precedenti, la riproduzione realistica degli oggetti, secondo un sitema di associazioni che rimanda in maniera simbolica alle sfere di competenza della defunta, deve intendersi come una sorta di certificazione dell'avvenuto assolvimento di queste.

Fig. 5 : Hydria apula a figure rosse

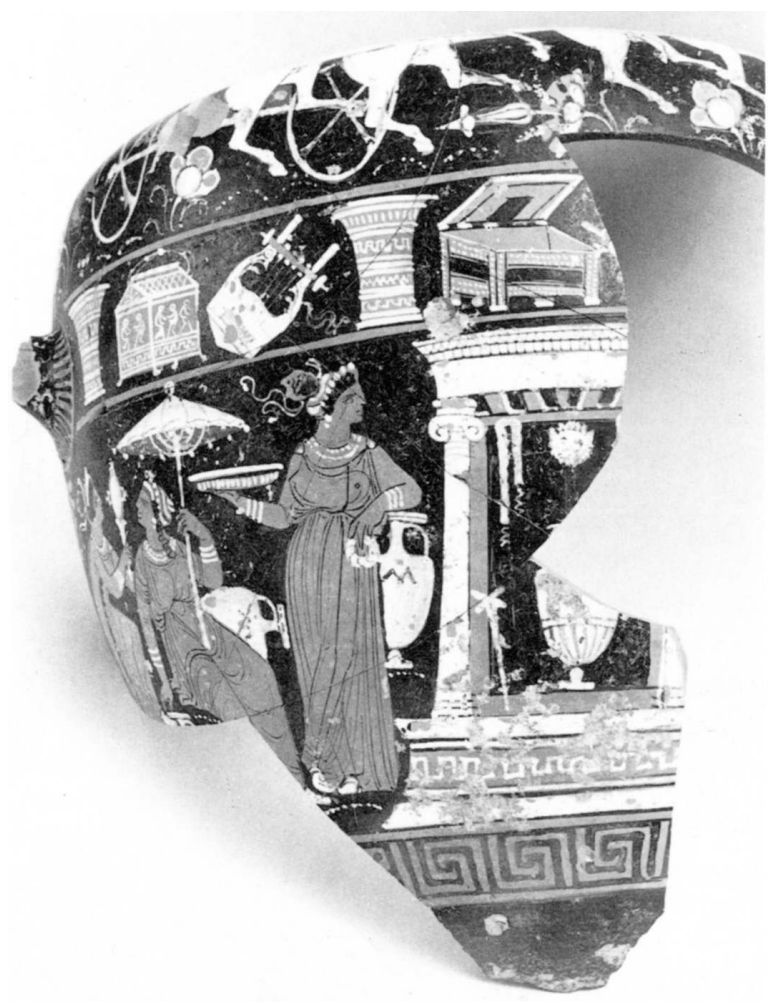

Den Haag, Collezione privata

SCHNEIDER-HERMANN 1961, fig. 1

Un'ulteriore conferma dell'interesse che il pittore di Baltimora nutre per la disposizione a fregio degli oggetti è offerta da un documento, purtroppo molto frammentario, appartenente ad una collezione privata dell'Aja ${ }^{69}$ (Fig. 5). Si tratta di un'hydria, vaso strettamente legato al mondo femminile e destinato anch'esso, come la loutrophoros, a contenere l'acqua, la cui decorazione è scandita al centro da un fregio, dove si dispongono in maniera paratattica: una coppia di kalathoi, una cista istoriata, una cetra, ed un 
cofanetto aperto, secondo un'associazione che già abbiamo visto documentata negli esemplari precedentemente analizzati. Se in questo vaso sembrerebbe prevalere una valenza ornamentale degli oggetti, proprio in virtù della posizione che occupano nella superficie decorata, tuttavia la loro presenza si può giustificare anche alla luce di quanto rimane delle decorazioni, la cui frammentarietà purtroppo non consente una lettura unitaria. Della parte superiore, rimangono tracce di un carro trainato da una coppia di cavalli ${ }^{70}$; meglio conservata è la parte inferiore del vaso, dove troviamo il motivo delle fanciulle alla fontana ${ }^{71}$. Rimangono tre donne, ciascuna appoggiata ad un'hydria e caratterizzata rispettivamente da uno specchio, un parasole ed una patera. L'ultima figura femminile volge lo sguardo verso un heroon, sul cui fondo si riconoscono: in alto una benda, in basso, appoggiata ad una colonna, una fiaccola a quattro bracci spenta, al centro ancora una grossa hydria con la parte inferiore baccellata, che riceve l'acqua da una fontana a protome leonina. La forte connessione che a livello iconografico si instaura tra fontana/acqua/hydriai dipinte indubbiamente amplifica il valore del contenitore ceramico che ne ospita la rappresentazione, in una sorta di raffinato gioco di rimandi iconografici che, come ha recentemente sottolineato François Lissarrague (LISSARRAGUE 2008), è abbastanza frequente nella ceramografia italiota e denuncia un uso consapevole da parte dell'artigiano delle potenzialità evocative dei segni iconografici. Il tema delle fanciulle alla fontana, come è noto, è rappresentazione dal forte contenuto simbolico che enuclea diversi livelli di significato tutti afferenti al tema dell'acqua, elemento fondamentale con le sue valenze purificatrici e fertilizzanti, nelle ritualità di passaggio, iniziazione e metamorfosi ${ }^{72}$. Se nel nostro caso il tema evocato, come probabile, è quello della lustratio funebre, come sembra suggerire anche la presenza della fiaccola a quattro bracci tradizionale attributo di Persefone nella sua valenza ctonia (PENSA 1977, p. 40), il simbolismo funerario connesso a questa rappresentazione trova corrispondenza nell'ordinata disposizione degli oggetti raffigurati nel fregio, che se da un lato alludono alle usuali offerte al morto, al contempo possono fungere da markers indispensabili a designare il ruolo della defunta, alla cui tomba il vaso era destinato.

Spunti ulteriori di riflessione emergono se allarghiamo lo sguardo e proviamo ad inserire le immagini che abbiamo visto sin qui nel più ampio quadro del linguaggio figurativo magnogreco coevo o leggermente anteriore, dove osserviamo come un dispositivo visuale per molti versi simile compare in alcuni vasi che mettono in scena la tragica vicenda di Niobe $^{73}$, un mito che viene scelto nelle comunità indigene come simbolo di una eroizzazione al femminile. Come è noto, si tratta della tragica vicenda della figlia del re di Lidia Tantalo e moglie del tebano Anfione che, orgogliosa dei suoi numerosi figli, sia maschi che femmine, osa vantarsi con insolenza su Latona, accusandola di aver generato solamente due figli, Apollo ed Artemide, i quali vendicano l'offesa rivolta alla madre, uccidendo tutti i Niobidi. Per l'immenso dolore Niobe si pietrifica, trasformandosi in roccia sul monte Sipilo. Marina MAZzEI (1999), in un importante contributo dal titolo Committenza e mito. Esempi dalla Puglia Settentrionale, ha messo ben in luce la rifunzionalizzazione regionale, in particolare nord-apula, di questo importante mito ${ }^{74}$. La vicenda di Niobe, come è noto, comincia ad essere attestato in ambito italiota in vasi destinati a cerimonie sepolcrali a partire dalla seconda metà del IV secolo a.C. ${ }^{75}$, in immagini che, con soluzione assai innovativa, selezionano il momento della pietrificazione dell'eroina, vera e propria mater dolorosa, icona della sofferenza e dell'afflizione, raffigurata isolata all'interno del naiskos ${ }^{76}$. 
Specificamente centrati sul personaggio femminile sono circa 9 vasi $^{77}$, quasi tutti di produzione apula tranne per un documento rinvenuto in Lucania (Roccagloriosa) ${ }^{78}$ ed uno di produzione campana ${ }^{79}$. In particolare per l'ambito apulo, gli studiosi hanno evidenziato una concentrazione di attestazioni figurate che va da Canosa ad Arpi, in un arco cronologico che si estende dal secondo all'ultimo quarto del IV a.C. ${ }^{80}$.

Fig. 6 : Loutrophoros apula a figure rosse

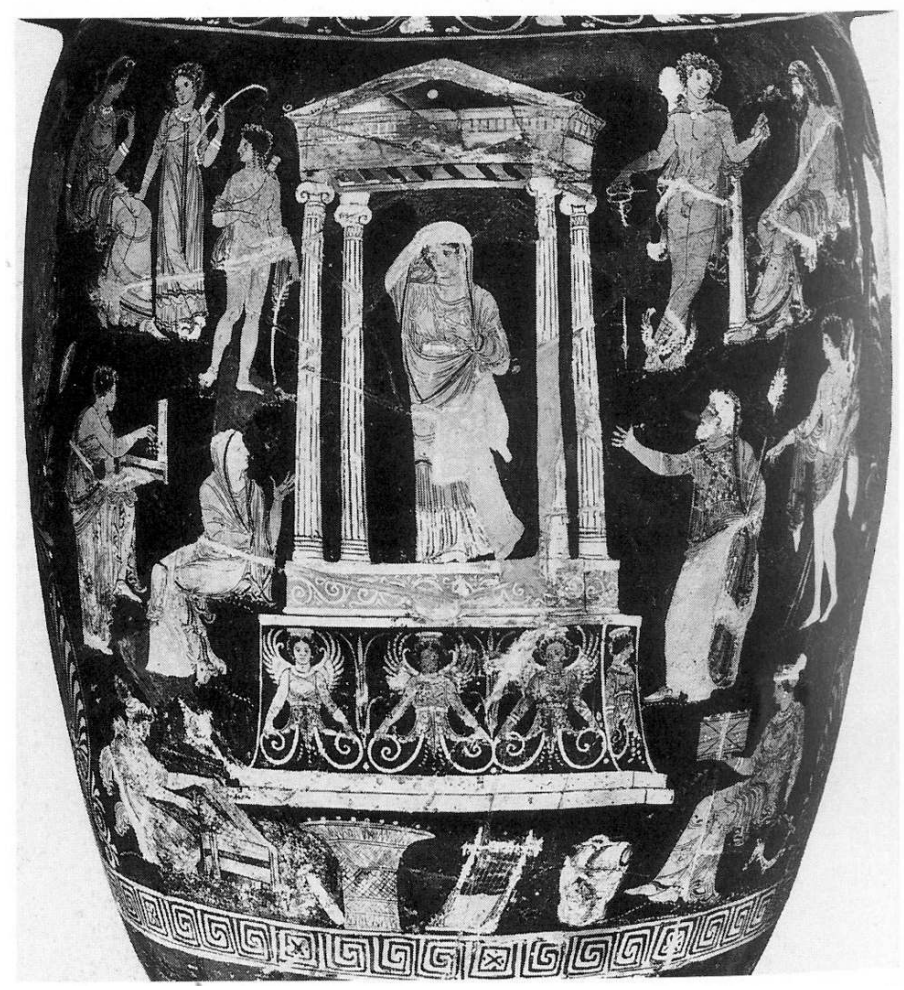

Napoli, Museo Archeologico Nazionale, inv. 82267

DE CESARE 1997, P. 286

Su una loutrophoros attribuita al pittore di Varrese e datata intorno alla metà del IV secolo a.C. ${ }^{81}$ (Fig. 6) Niobe è raffigurata all'interno di un elegante naiskos ${ }^{82}$, colta nel tipico gesto di lutto; la presenza del colore bianco nella parte inferiore del corpo è un dettaglio con cui il pittore ha voluto comunicare all'osservatore la graduale pietrificazione in atto ${ }^{83}$. Intorno al naiskos si dispongono numerose figure in tre registri sovrapposti: in alto possiamo riconoscere i protagonisti divini del mito: a sinistra, Latona con Artemide ed Apollo; a destra Hermes e Zeus ${ }^{84}$. Nel secondo registro, si potrebbero invece riconoscere seguendo l'interpretazione di CH. AELLEN $(1994$, p. 70$)$ - i personaggi della famiglia : nella donna velata a sinistra del naiskos la madre dell'eroina oppure la nutrice dei figli di Niobe ${ }^{85}$; a destra, nel vecchio con barba bianca che compie un evidente gesto di supplica, si può riconoscere il padre di Niobe, Tantalo, a sua volta figlio di Zeus ${ }^{86}$, affiancati rispettivamente da una giovane donna e da un giovane uomo; nel registro inferiore due giovani donne, entrambe raffigurate con un cofanetto in mano. La scelta di usare nel corredo funerario un vaso che raffigura Niobe circondata dal suo gruppo familiare viene letta da alcuni studiosi come una precisa volontà di auto-rappresentazione da parte della defunta $^{87}$. Secondo questa prospettiva, prenderebbe senso anche la raffigurazione della parte inferiore del vaso dove, al di sotto del naiskos, deposti lungo la linea del meandro, 
sono dipinti una serie di oggetti, secondo le modalità che ritroveremo più tardi nei vasi del pittore di Baltimora: un alabastron per gli unguenti, un kalathos con dentro uova o frutti, una lira ${ }^{88}$ ed una corazza anatomica ${ }^{89}$. Abbiamo in questa immagine una serie di oggetti significanti ${ }^{90}$, che acquistano una notevole forza segnaletica in virtù di un loro statuto, differente da quello degli altri oggetti che entrano nel gioco delle relazioni (perché tenuti in mano oppure offerti). In particolare, in questo vaso risulta di un certo interesse l'associazione di alabastron, kalathos, lira e corazza, oggetti che mettono in gioco i diversi ruoli dell'uomo e della donna: se l'alabstron come il kalathos possono rimandare al genere femminile e ad un contesto domestico, al mondo maschile rinviano da un lato la lira, strumento polisemantico che rimanda sia alla paideia giovanile e alla pratica sociale del simposio ${ }^{91}$, dall'altro la corazza ${ }^{92}$, oggetto quest'ultimo che trova confronti nei realia delle tombe dell'Apulia centrale ${ }^{93}$, da leggersi in entrambi i contesti come simbolo per eccellenza di una posizione elitaria maschile affidata alla virtus guerriera ${ }^{94}$. In quest'immagine ritengo sia interessante la complementarietà di segni maschili e femminili, messa in luce attraverso una precisa selezione di oggetti semanticamente legati tra loro, che potrebbe forse suggerire la volontà di evocare un sistema familiare, collegato alla donna esaltando il tema, nodale nelle società arcaiche, della discendenza, che certifica la virtus femminile all'interno dell'istituto matrimoniale ${ }^{95}$.

Fig. 7 : Loutrophoros apula a figure rosse

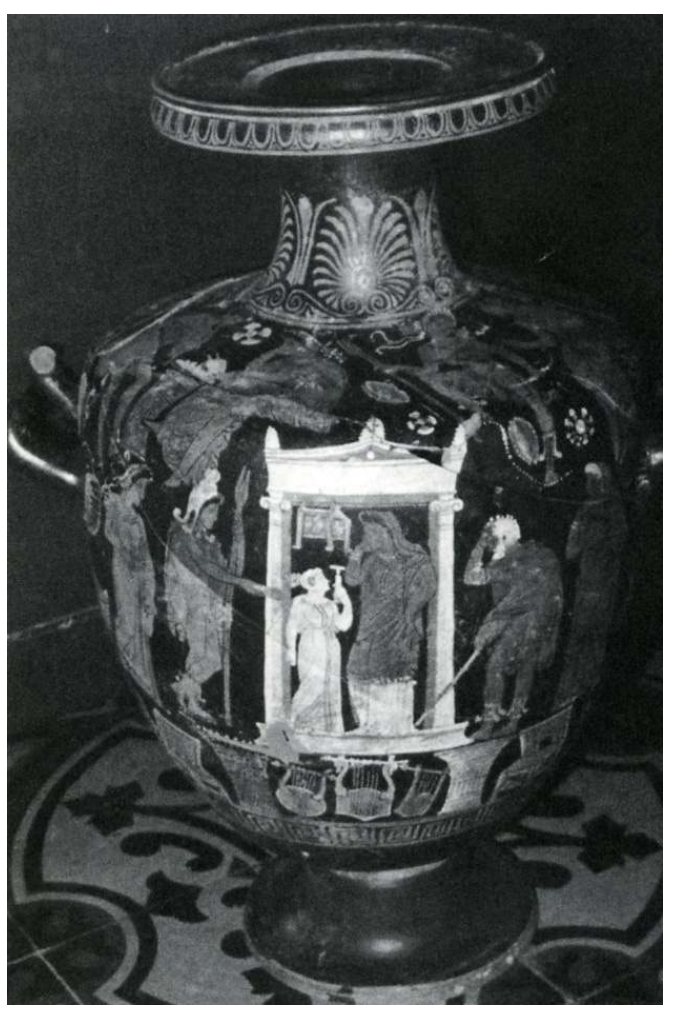

Zurich, Archäologische Sammlung der Universität 4007

dE CESARE 1997, P. 286

Il medesimo sistema di oggetti lo ritroviamo sull'hydria attribuita dal Trendall al pittore di Ganimede (Fig. 7) (340-320 a.C.) ${ }^{96}$. Il centro focale dell'immagine è occupato dalla figura di Niobe, riconoscibile all'interno del naiskos per i ritocchi di bianco sul fondo della veste che rivelano la metamorfosi in atto; accanto a lei è raffigurata una fanciulla (forse una 
Niobide ?), anch'essa tutta dipinta di bianco, resa mentre regge un alabastron ${ }^{97}$. Sopra la testa della fanciulla, sospeso nel campo dell'immagine, è raffigurato un cofanetto istoriato. Anche in quest'immagine, una sequenza di oggetti isolati, non manipolati e di dimensioni superiori agli altri, sono disposti paratatticamente sulla stessa linea in corrispondenza dello spazio del naiskos sotto la tomba: qui, in particolare tre lire sono inquadrate da due cofanetti e due kalathoi.

Fig. 8 : Hydria campana a figure rosse

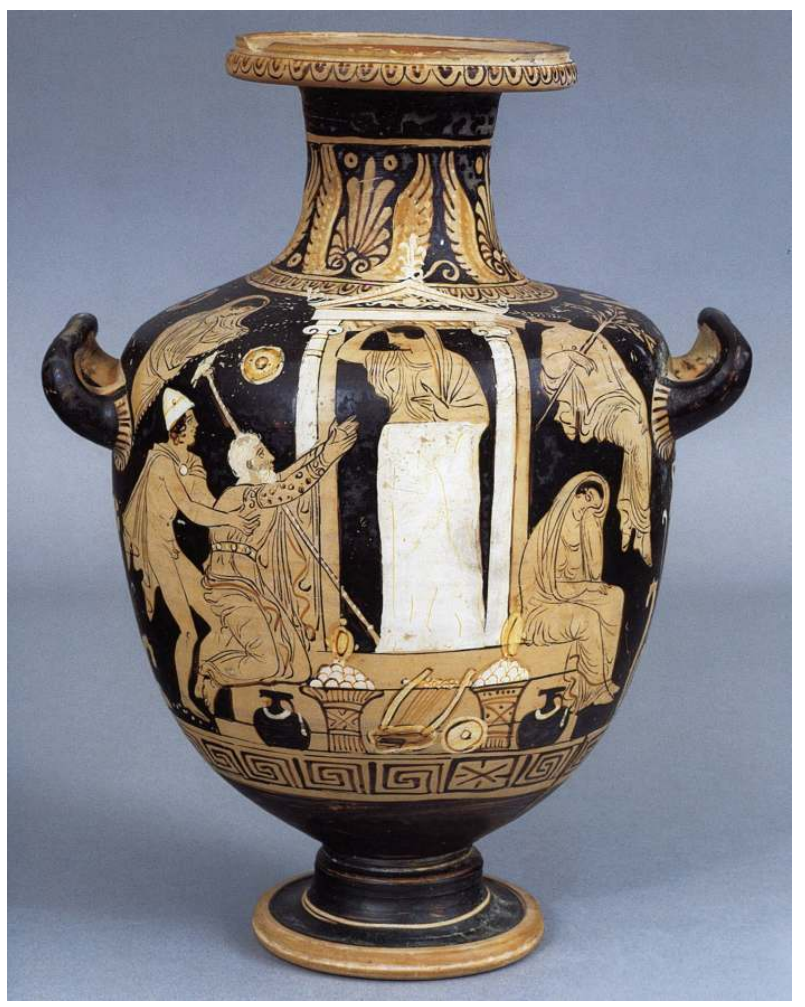

SYDNEY, THE NICHOLSON MUSEUM, INV. N. 71.01

SCHMIDT 1992, N. 11

La fortuna di tale sistema decorativo in area magnogreca viene confermata anche da esempi esterni al mondo apulo. Mi riferisco ad un'hydria di produzione campana (Fig. 8) $(340-330 \text { a.C. })^{98}$, dove Niobe entro l'edicola funeraria mostra una pietrificazione più avanzata: in questo vaso attribuito dal Trendall al pittore della Libagione, troviamo appoggiati davanti ai gradini del naiskos, in ordinata disposizione paratattica, una coppia di hydriai ed una coppia di kalathoi con frutti (o uova), sormontati entrambi da una patera, una lira al centro ed ancora una patera, secondo una soluzione formale che nell'integrazione tomba/oggetti, sembra maggiormente riecheggiare l'immaginario attico, ispirandosi alle scene di visita alla tomba delle lekythoi a fondo bianco ${ }^{99}$.

24 I legami più o meno espliciti tra i prodotti del pittore di Baltimora e le soluzioni iconografiche adottate per rendere il mito di Niobe, fissato nelle immagini apule nello stesso arco cronologico e nel medesimo ambito territoriale, sembrerebbero suggerire il prevalere di un preciso "gusto" della committenza apula, ben consapevole nel selezionare forme e contenuti al fine di esaltare l'aretè femminile ${ }^{100}$. 
orza propositiva del modello mitico viene dunque ripresa dal pittore di Baltimora, che struttura la decorazione dei vasi, sin qui analizzati, secondo un meditato codice visivo di comunicazione: se il focus è costituito dalla donna raffigurata entro il naiskos, motivo iconografico già di per sé eroizzante e caro alle aristocrazie indigene della fine del secolo, questo dialoga e si integra con la selezione e la particolare disposizione degli oggetti nel campo dell'immagine, cui il pittore affida un essenziale ruolo comunicativo. Specchi, pissidi, alabastra, cassettine, kalathoi, palle, oggetti tutti più o meno riconducibili al mundus muliebris e spesso presenti anche tra i realien dei corredi, non sono presentati come insieme funzionale: isolati, non manipolati ma, nello stesso tempo semanticamente legati tra loro, funzionano come simboli di un codice condiviso e strutturano un consapevole sistema di segni attraverso il quale comunicare informazioni sullo status, il ruolo sociale della defunta rappresentata e la sfera di valori connessa.

\section{BIBLIOGRAFIA}

AELLEN CH. (1994), À la recherche de l'ordre cosmique. Forme et fonction des personnifications dans la céramique italiote, Kilchberg.

ANDREASSI G. (1979), Ceramica italiota a figure rosse della Collezione Chini del Museo Civico di Bassano del Grappa, Roma.

BAGGIO M. (2004), I gesti della seduzione. Tracce di comunicazione non verbale nella ceramica greca tra VI $e$ IV secolo a.C., Roma.

BAGGIO M. (2008), Il mondo al femminile nel repertorio figurativo apulo. La prospettiva degli oggetti, in Vasi immagini collezionismo 2008, p. 285-310.

BAGGIO M., SALVADORI M. (2011), Maschile-femminile nella pittura funeraria ellenistica: tra rmi ed ornamento. Per una dialettica spazio-oggetto, dans La Torre G. F., Torelli M. éd., Pittura ellenistica in Italia e in Sicilia. Linguaggi e tradizioni, Roma, p. 79-90.

CASSANO R. (1992), Ceramica a figure rosse, dans Cassano R. éd., Principi, Imperatori Vescovi : duemila anni di storia a Canosa, Venezia, p. 261-301.

CASSANO R. (1996), Ruvo, Canosa, Egnatia e gli scavi dell'Ottocento, dans Pugliese Carratelli G. éd., I Greci in Occidente. La Magna Grecia nelle collezioni del Museo Archeologico di Napoli, Napoli, p. 108-113.

CAssimatis H. (1990), Propos sur le calathos dans la céramique italiote, dans Descoeudres J-P. éd., Eumousia. Ceramic and Iconographic Studies in Honour of Alexander Cambitoglou, Sydney, p. 197-205.

CASsimatis H. (1995a), Cosmétique et funéraire sur les vases apuliens, dans di Marchegay S., Le Dinahet M.-Th., Salles J.-F. éd., Nécropole et pouvoir. Idéologies, pratiques et interprétations, Actes du colloque « Théories de la nécropole antique », Lyon, p. 155-166.

CASSIMATIS H. (1995b), « Fenêtre de l'au-delà dans l'iconographie italiote », MEFRA 107, p. 1061-1092.

CASSIMATIS H. (1998), « Le miroir dans les représentation funéraires apuliennes », MEFRA 110, 1, p. 297-350. 
CASsimatis H. (2000), «L'empire des signes » : les vases italiotes, dans Cavalier O. éd., Terres sacrées de Perséphone : collections italiotes du Musée Calvet- Avignon, Paris, p. 39-49.

Céramique apulienne (2005), DENOYelle M., LipPolis E., MAZZEI M., POUZAdoux C. éd., (La) Céramique apulienne. Bilan et perspectives, Napoli.

CHIECo-BIANCHi MARTINi A.M. (1964), « Conversano (Bari) - Scavi di via T. Pantaleo », Notizie degli Scavi di Antichità VIII, 18, p. 100-176.

CIANCIO A. 1997, Silbíon. Una città tra Greci e indigeni, Bari.

Colivicchi F. (2004), «L'altro vino. Vino, cultura e identità nella Puglia e nella Basilicata anelleniche », Siris 5, p. 23-68.

Colivicchi F. (2006), Lo specchio e lo strigile. Scambio di simboli e scambio tra i sessi, dans Massa-

Pairault F.-H. éd., L'image antique et son interprétation, Roma, p. 277-300.

COLIVICCHI F. (2009), Warriors and citizens. Models of self-representation in native Basilicata, dans

Osanna M. éd., Verso la città Forme insediative in Lucania e nel mondo italico tra IV e III sec. a.C.,Venosa, p. 69-88.

CORRENTE M. (2003), Canusium. L'ipogeo dei serpenti piumati, Bari.

CORRENTE M. (2005), La ceramica a figure rosse a Canosa e nel territorio : $i$ dati delle recenti scoperte, dans Céramique apulienne, p. 59-76.

Cultura e religione delle acque (2012), CALDERONE A. éd., Cultura e religione delle acque, Roma.

DE CESARE M. (1997), Le statue in immagine : studi sulle raffigurazioni di statue nella pittura vascolare greca, Roma.

DENOYELLE M. (1997), Le cratère des Niobides, Paris.

DENOYELLE M., IOZZO M. (2009), La céramique grecque d'Italie Méridionale et de Sicile, Paris.

DOLCI M. (2004), Ceramica apula a figure rosse. La produzione apula tarda, dans Sena Chiesa G. éd., La Collezione Lagioia : una raccolta storica della Magna Grecia al Museo archeologico di Milano, Milano, p. 153-238.

ESPOSITO A., PEDRINA M. (2003), Autour du naiskos, dans Griechische Keramik 2003, p. 182-184.

FONTANNAZ D. (2008), «L'entre-Deux-Monde : Orphée et Eurydice sur une hydrie proto-italiote du sancyuaire de la source à Saturo ", $A K$ 51, p. 41-72.

FRONTISI-DUCROUX F. (2003), L'homme-cerf et la femme araignée, Paris.

FRONTISI-DUCROUX F., VERNANT J.-P. (1998), Ulisse e lo specchio. Il femminile e la rappresentazione di sé nella Grecia antica, Roma.

GERNET L. (1948), « La notion mythique de la valeur en Grèce », Journal de Psychologie 41, p. 415-462.

GERNET L. (1968), Anthropologie de la Grèce antique, Paris.

GHERCHANOC F., HUET V. (2009), « S'habiller et se déshabiller en Grèce et à Rome (I). Pratiques politiques et culturelles du vêtement. Essai historiographique », Revue historique CCCIX/I, p. 3-30. GIACOBELLO F. (2008), Lo spazio interno nella ceramica apula, dans Vasi immagini collezionismo 2008, Milano, p. 267-284.

Griechische Keramik (2003), Griechische Keramik im kulturellen Kontext, Akten des Internationalen Vasen-Symposions (Kiel vom 24. Bis 28.9.2001), Münster. 
GUALTIERI M. (2003), Élites lucane ed immagini: Niobe a Roccagloriosa, dans Giudice F., Panvini R. éd., Il greco, il barbaro e la ceramica attica. Immaginario del diverso, processi di scambio e autorappresentazione degli indigeni,Atti del Convegno Internazionale di Studi, Catania, p. 147-153.

GUALTIERI M. (2005), Discussion, in Céramique apulienne 2005, p. 216-219.

GUALTIERI M. (2006), La committenza della ceramica a figure rosse tardo-apula: un caso di studio, dans de la Genière J. éd., Les clients de la céramique grecque, Actes du Colloque de l'Académie des Inscriptions et Belles Lettres, Paris, p. 99-106.

GUZzo P. G. (1993), L'armamento in Lucania tra IV e III secolo a.C., dans Bottini A. éd., Armi. Gli strumenti della guerra in Lucania, Bari, p. 157-174.

HALM-TISSERANT M. (2004), « “Keimenon” : de l'objet réifié à l'objet “sujet” dans la peinture de vases grecques », Pallas 65, p. 33-48.

HALM-TISSERANT M. (2006), « Symbolique et étique du blanc e du noir dans la pensée et dans l'imagerie en Grèce ancienne », Eidola. International journal of classical art history 3, p. 9-28.

ISLER KERÉNYI C. (2004), Dioniso ed Eros nella ceramica apula, dans Miti greci 2004, p. 244-250.

KEULS E. (1978), « Aeskylus' Niobe and apulian funerary symbolism», ZPE 30, p. 41-68.

KOKULA G. (1983), Marmorloutrophoren, Berlin.

LAMBRUGO Cl. (2004), Lusso e raffinatezza nella moda femminile di IV secolo a.C., dans Miti greci 2004, p. 205-206.

LIPPOLIS E. (1994), La tipologia dei semata, dans Lippolis E. éd., Taranto. La necropoli: aspetti e problemi della documentazione archeologica dal VII al I secolo a.C., catalogo del Museo Nazionale Archeologico di Taranto, III, 1, Taranto, p. 239-281.

LISSARRAGUE F. (1995), Women, boxes, containers: some signs and metaphors, in Reeder E. éd., Pandora. Women in classical Greece, Baltimore-Princeton, p. 91-101

LISSARRAGUE F. (2006), « De l'image au signe. Objets en représentation dans l'imagerie grecque», Quoi de neuf en histoire ancienne ?, Cahiers du centre de recherches historiques 37, p. 11-23.

LISSARRAGUE F. (2008), Réflexions sur l'image dans la céramique de Grande Grèce, dans Vasi immagini collezionismo, 2008, p. 209-228.

LOHMANN H. (1979), Grabmäler auf unteritalishen Vasen, Berlin.

MASSA-PAIRAUlT F.-H. (2009), Philippos Laos, dans Volpe G., Strazzulla M.J., Leone D. éd., Storia e archeologia della Daunia, in ricordo di Marina Mazzei, Bari, p. 195-204.

MAZZEI M. (1990), « L’ipogeo Monterisi Rossignoli di Canosa », AION (archeol) 12, p. 123-167.

MAZZEI M. (1999), Committenza e mito. Esempi dalla Puglia settentrionale, dans Massa Pairault F.-H. éd., Le mythe grec dans l'Italie antique. Fonction et image, Roma, p. 467-483.

MENICHETTI M. (2006), La donna alla fontana. Charis e matrimonio sulle ciste prenestine, dans Colpo I., Favaretto I., Ghedini F. éd., Iconografia 2005. Immagini e immaginari dall'antichità classica al mondo moderno, Roma, p. 51-64.

Miti greci (2004), SENA CHIESA G., ARSLAN E. éd, Miti greci Archeologia e pittura dalla Magna Grecia al collezionismo, catalogo della mostra, Milano.

MÖsCH R.-M. (1988), «Le mariage et la mort sur les loutrophores», AION (archeol) 10, p. 117-139. 
MösCH R.-M. (2006), Die loutrophoros im Hochzeits-und Begräbnisritual des 5. Jahrhunderts v. Chr. in Athen, Berne.

MONTANARO A. (2007), Ruvo di Puglia e il suo territorio: le necropoli. I corredi funerari tra la documentazione del XIX secolo e gli scavi moderni, Roma.

MORARD TH. (2009), Horizontalité et verticalité. Le bandeau humain et le bandeau divin chez le Peintre de Darius, Mainz am Rhein.

MORET J.-M. (1978), « Le Jugement de Pâris en Grande-Grèce : mythe et actualité politique ", AK 21, p. 76-98.

MORET J.-M. (1984), Oedipe, la spinx et les Thébains. Essai de mythologie iconographique, Genève.

Mugione E. (2000), Miti della ceramica attica in Occidente. Problemi di trasmissioni iconografiche nelle produzioni italiote, Taranto.

NEILS J. (2008), Women are white. White ground and the attic funeral, dans Lapatin K. éd., Papers on Special Tecniques in Athenian vases, p. 61-72.

NIDDAN N., STRAWCZYNSKI N. (2003), Objets identifiants. La Lyre, dans Griechische Keramik 2003, p. 157-159.

OAKLEY J.H. (2004), Picturing death in classical athens : the evidence of the white lekythoi, Oxford.

(Le) peintre de Darius et son milieu (1986), AELLEN Ch., CAMBIToglou A., CHAMAY J. éd., Le peintre de Darius et son milieu: vases grecs d'Italie méridionale, Genève.

PENSA M. (1977), Rappresentazioni dell'oltretomba nella ceramica apula, Roma.

PICAUD S. (2004), «Les représentations de jeux de balles et des osselets dans les terres cuites, céramiques et reliefs ", Pallas 65, p. 49-55.

PONTRANDOLFO A. et al. (1988), « Semata e Naiskoi nella ceramica italiota », AION(archeol) 10, p. 181-202.

PONTRANDOLFO A. (1988), L'escatologia popolare e i riti funerari greci, dans Pugliese Carratelli G. éd., Magna Grecia, III. Vita religiosa e cultura letteraria, filosofica e scientifica, Milano, p. 171-185.

PONTRANDOlFo A., ROUVERET A. (1992), Le tombe dipinte di Paestum, Modena.

POUZADOUX cl. (2005), L'invention des images dans la seconde moitié du IV siècle : entre peintres et commanditaires, dans Céramique apulienne 2005, p. 187-200.

ROBINSON E.G.D. (1990), Workshops of Apulian Red-Figure outside Taranto, dans Descoeudres J.-P. éd., Eumousia. Ceramic and Iconographic Studies in Honour of Alexander Cambitoglou, Sydney, p. 181-197.

RICCARDI A. (2003), Gli antichi Peucezi a Bitonto. Documenti ed immagini dalla necropoli di via Traiana, Bari.

RICCARDI A. (2008), Donne e guerrieri da Ruvo e Bitonto. Le scoperte del III millennio, Bari.

ROUVERET A. (1990), Tradizioni pittoriche magno greche, dans Pugliese Carratelli G. éd., Magna Grecia arte e artigianato, Milano, p. 317-349.

RVAP I= TRENDALL A. D., CAMBITOGLOU A. (1978), The red-figured vases of Apulia. I. Early and middle apulian, Oxford.

RVAp II= TRENDALL A. D., CAMBITOGLOU A. (1982), The red-figured vases of Apulia. II. Late apulian, Oxford. 
RVAp, Suppl I = TRENDALL A. D., CAMBITOGLOU A. (1991), Second supplement to The red-figured vases of Apulia. Part I. Chapters 1-20, London (BICS 60).

RVAp, Suppl I = TRENDALL A. D., CAMBITOGLOU A. (1992), Second supplement to The red-figured vases of Apulia. Part II. Chapters 21-30, London (BICS 60).

SABETAI V. 2009, Loutrophoroi in context, dans Tsingarida A. éd., Shapes and Uses of Greek Vases $\left(7^{\text {th }}-4^{\text {th }}\right.$ centuries B.C.), Bruxelles, p. 291-306.

SCHAUENBURG K. (2000), Studien zur unteritalischen Vasenmalerei, II, Kiel.

SCHMIDT M. (1992), Niobe, dans LIMC, VI, p. 908-914.

schmidT M. (1996), La ceramica italiota e siceliota, dans Pugliese Carratelli G. éd., I Greci in Occidente, Catalogo della mostra, Venezia, p. 443-456.

SCHNEIDER-HERMANN G. (1961), « Ein apulisches Vasenfragment », BABesch 36, p. 64-70

SCHNEIDER-HERMANN G. (1971), « Der Ball bei den Westgriechern », BABesch 46, p. 123-133.

SCHNEIDER-HERMANN G. (1975), « Katalog », BABesch I Suppl., p. 50-51.

SENA CHIESA G. (2006), Il periodo apulo tardo : dal mondo del mito al mondo degli affetti, in SENA CHIESA G. éd., Ceramiche attiche e magnogreche. Collezione Banca Intesa, Milano, p. 386-395.

SMITH H.W.R. (1970), « Deadlocks ? », BABesch 45, p. 68-85.

SMITH H.W.R. (1976), Funerary symbolism in apulian vase painting, California.

TODisCo L., CATUCCI M. (2007), Museo Nazionale di Matera « Domenico Ridola »-Collezione Rizzon, CVA

Italia LXIII, Museo nazionale di Matera, I, p. 63-65, tav. 69, 1-4.

TODIsco L. (2008), Il pittore di Arpi: mito e società nella Daunia del tardo IV secolo a.C., Roma.

TRENDALL A.D. (1985), An Apulian loutrophoros representing the Tantalidae, Greek vases in the J. Paul Getty Museum 2, p. 129-149.

TRENDALL A.D. (1989), The red-figured vases of South Italy and Sicily. A handbook, London.

Vasi immagini collezionismo (2008), SENA CHIESA G. éd. (2008), Vasi immagini collezionismo. La collezione di vasi Intesa Sanpaolo e i nuovi indirizzi di ricerca sulla ceramica greca e magnogreca, Milano.

\section{NOTE}

1. Numerosi spunti di riflessione vengono soprattutto dai lavori che affrontano il grande tema della selezione e diffusione, nella seconda metà del IV secolo a.C., di « miti al femminile », quali Niobe, Andromeda, Danaidi, Europa, molto importanti anche per la valenza purificatrice che riflettono in ambito funerario. Per una sintesi si vedano : MAZZEI 1999, p. 467-483 e POUZADoux 2005, p. 187-200.

2. Per un'indagine sul significato degli oggetti femminili nell'immaginario figurato della pittura funeraria ellenistica si veda BAGGIO, SALVADORI 2011.

3. Il contributo che qui si presenta si inserisce all'interno di una più ampia ricerca dedicata allo studio del significato che determinati oggetti acquisiscono nel momento in cui entrano in relazione col mondo femminile, in particolare nelle immagini vascolari attiche e magnogreche. Si vedano in proposito i contributi contenuti in due numeri della rivista Pallas (il numero 63 del 2003 ed il numero 65 del 2004), che dedicano una specifica sezione all'objet dans l'image.

4. POI GERNET 1968. 
5. Sui significati che può assumere lo specchio in seno alla comunità antica e sul suo valore in relazione al percorso di erotizzazione femminile si vedano anche i lavori di Mauro MENICHETTI (in particolare 2006, con bibliografia). Importanti riflessioni anche in COLIVICCHI 2006.

6. LISSARRAGUE 1995, p. 91.

7. Si veda quanto scritto recentemente da C. ISLER KÉRENYI (2004), in relazione ad esempio alla frequente comparsa dei medesimi oggetti in serie tematiche diverse, legate al mondo ora di Afrodite, ora di Eros, ora di Dioniso ora all'ambito funerario. Poiché nella ceramica italiota queste sfere tematiche non sono mai ermeticamente separate, ma l'una finisce per fondersi nell'altra, di conseguenza gli oggetti che entrano nel campo di queste divinità finiscono per caricarsi di un valore ambiguo, definibile di volta in volta solo in base al contesto ed alle associazioni.

8. Concetto ben espresso in MORET 1984, p. 156 : « ...voir, c'est reconnaître, c'est-à-dire identifier la figure ou l'objet en function de ce qu'on sait déjà ».

9. La studiosa ha ben evidenziato la vasta gamma di significati messi in campo dallo specchio nella ceramica italiota: è oggetto-simbolo dell'universo femminile ma, al tempo stesso, veicola significati magici e religiosi : lo specchio è strumento in grado di aprire verso una dimensione altra dell'esperienza visuale e di favorire la comunicazione tra il mondo dei vivi e quello dei morti.

10. CAssimatis 2000, p. 47 : « un thyrse ne signifie pas que l'homme qui le tient est Dionysos, mais que le contexte est dionysiaque ».

11. RVAp II e RVAp suppl. II, p. 268-291.

12. Sulla bottega del pittore di Dario si veda (Le) peintre de Darius et son milieu 1986.

13. DENOYELLE, IOZZO 2009 , p. 158 . La produzione del suo atelier offre un ampio ventaglio tipologico : 34 crateri a volute, 1 cratere a colonnette, 11 anfore, 8 loutrophoroi, 12 hydriai, 2 lebetes gamikoi, 6 kantharoi, 6 pissidi, 9 piatti, 3 vasi plastici, 1 skyphos, 15 oinochoai, cui si aggiungono numerose lekanidi ed alcune patere. Per la produzione del pittore di Baltimore a Canosa si veda anche : CORRENTE 2005, p. 72-73. Nessun esemplare, almeno sino al momento sembra essere stato trovato nell'area tarantina.

14. Si veda in proposito ROBINSON 1990.

15. A partire dalla metà del IV secolo a.C. si ha un'importanza crescente della committenza rappresentata dalle élites indigene ellenizzate, che in questo periodo sembrano maggiormente emergere in virtù di interessi agropastorali e che le rende capaci di acquisire maggiormente beni : cf. DOLCI 2004, p. 154.

16. Secondo Marisa CORRENTE (2005, p. 69), in questo periodo appare una strutturazione di gruppi egemoni fortemente permeati da una volontà di auto-rappresentazione e che hanno completamente appreso quelle che sono le possibilità di significazione delle immagini, in particolare dei miti.

17. Che conta, secondo il catalogo del Trendall, più di un centinaio di pezzi.

18. Taranto 9847 (RVAp II, p. 869, n. 44); Foggia 132738, da Arpi (RVAp II, p. 869, n. 45); Amburgo, collezione Termer (RVAp II, p. 869, n. 45a); Louvre S 4053 (RVAp II, p. 869, n. 46) Fiesole, Collezione Costantini 154 (RVAp II, p. 869, n. 47) ; Basilea Mercato antiquario (RVAp II, p. 869, n. 48) ; Roma Villa Giulia 15691 (RVAp II, p. 869, n. 49); Malibu 77 AE16 (RVAp II, p. 869, n. 49a) ; Matera, Museo Ridola.

19. La loutrophoros nasce nel mondo greco e, accanto ad un uso funerario ben attestato, aveva una prevalente funzione nuziale, come recipiente usato dalla giovane sposa per il bagno rituale. Tale destinazione è confermata dal ritrovamento in grande quantità di loutrophoroi sul versante meridionale dell'Acropoli, in una zona epigraficamente individuata come Santuario delle Ninfe. Si veda da ultimo anche SABETAI 2009. Sulle loutrophoroi attiche anche KоKULA 1984; мӧSСН 1988 ; MÖSCH 2006. 
20. Si vedano in proposito: ANDREASSI 1979 ; PONTRANDOLFO et alii 1988, p. 185 ; SCHAUENBURG 2000, p. 19-21; DOLCI 2004, con bibliografia precedente; FONTANNAZ 2008.

21. RVAp, Suppl. II, p. 281, n. 48e; CASSIMATIS 2000.

22. Il motivo entra a far parte dell'iconografia vascolare apula intorno alla metà del IV secolo a.C. per svilupparsi più ampiamente nella seconda metà del secolo: sul tema, sempre utile PONTRANDOLFO et alii 1988. Per un repertorio di riferimento : cf. LOHMANN 1979 ; per un rapporto con i realia rinvenuti a Taranto : cf. LIPPOLIS 1994.

23. Sull'altro lato del vaso, come di consueto nella produzione di questo pittore, troviamo una versione più semplificata di scena con defunta entro naiskos : qui una figura femminile seduta su roccia tiene in mano una cassettina ed una corona.

24. LAMBRUGO 2004, p. 205. In numerose tombe apule di IV secolo a.C. il prestigio sociale e il ruolo di rilievo rivestito dalla defunta in seno alla comunità di appartenenza viene sottolineato attraverso l'offerta nella tomba di elementi propri dell'ornamento personale, quali collane, orecchini, vaghi in materiali anche preziosi : cf. RICCARDI 2003, p. 101.

25. Abbastanza inconsueta la postura della donna stante a gambe incrociate, che trova però un interessante antecedente nella raffigurazione di una loutrophoros apula attribuita alla Cerchia del Pittore di Gioia del Colle, ora proprietà Banca Intesa (DOLCI 2004, p. 372).

26. Su questa loutrophoros si veda anche ESPOSITO, PEDRINA 2003; sui possibili significati del parasole rimando anche BAGGIO 2008, con bibliografia precedente (in partic. p. 285-310). Nell'ambito della produzione del pittore di Baltimora il parasole compare assai frequentemente: come attributo femminile (Baltimore 48.86, dans RVAp II, 27/21, pl.324); in mano alla dea Afrodite (Foggia 132732, dans RVAp II, 27/28, pl. 326, 1) ; in mano ad un'ancella (Bari, Collezione de Blasi Cirillo, dans RVAp II, 27/40, pl.327); in mano ad offerenti in scene di naiskos (Fiesole, Collezione Costantini 152 e 153, Mattinata, Collezione Sansone 685; Bari, Collezione Macinagrossa 26, dans RVAp II, 27/54-57, pl. 331, 1-4).

27. Insistono sul particolare gesto ESPOSITO, PEDRINA 2003.

28. Sul legame tra il colore bianco ed il corpo femminile si veda, da ultimo, NEILS 2008.

29. Nell'uso del colore aggiunto non manca, inoltre, il riferimento alla nuova realtà spirituale del defunto che partecipa della sfera ultraterrena ed, al contempo, di una sorta di « trafigurazione ». Sui valori simbolici del colore bianco si veda HALM-TISSERANT 2006.

30. Sullo spazio interno nella ceramica apula si veda GIACOBELLO 2008, p. 267-284.

31. ESPOSITO, PEDRINA 2003, p. 182.

32. In alto, a sinistra, una figura femminile seduta regge con una mano una cista lavorata con manico, con l'altra una cassettina (forse di vimini intrecciato) ed una balla di lana ; in basso, una donna stante regge un ventaglio ed una oinochoe rovesciata ; in alto, a destra, ancora una figura femminile seduta regge un ventaglio ed una cista; in basso, a destra, una donna regge uno specchio ed una situla.

33. Sulla difficoltà di conoscere l'identità dei personaggi intorno al naiskos e la loro relazione col defunto e il suo mondo, si veda anche SCHMIDT 1996 e, da ultimo, MASSA-PAIRAULT 2009, p. 196.

34. Riprendo il termine da PONTRANDOLFo, ROUVERET 1992.

35. Su questi oggetti CASSIMATIS 1995a.

36. CASSIMATIS 2000.

37. Si veda anche BAGGIO 2004, p. 141 ss.

38. Ricordiamo che Elena, nell'Odissea (Omero, Od. IV, 131 ss.), reintegrata nel ruolo di sposa, siede accanto a Menelao con i doni bellissimi che le diede la sposa di Polibio : una conocchia d'oro ed un cesto d'argento munito di ruote con $i$ bordi dorati (trad. M. G. Ciani).

39. Kalathoi miniaturistici compaiono, ad esempio, in corredi femminili apuli databili alla metà del IV secolo a.C. rinvenuti a Conversano, nelle tombe $6,8,9$, per cui si veda CHIECO-BIANCHI MARTINI 1964. Kalathoi a decorazioni geometriche sono attestati tra i realia in tombe apule 
rinvenute a Silbíon (nella tomba 2/1967 e nella tomba 2/1994, per cui si veda CIANCIO 1997, p. 197, n. 186 e p. 206, n. 226). In particolare, nella tomba 3/1994 rinvenuta sempre a Silbíon (CIANCIO 1997, p. 224, nn. 291 e 292), il kalathos è associato a parti bronzee di un cofanetto ligneo.

40. Attività testimoniata anche dal rinvenimento, in contesti tombali femminili, di pesi da telaio : si veda, ad esempio, il peso da telaio rinvenuto nella tomba 3/2002 della necropoli di Bitonto, letto come « esplicito riferimento alla tessitura praticata dalle donne dell'antichità » : si veda Ada RICCARDI 2008 , p. 17 , fig. 31 ; oppure il peso da telaio rinvenuto nella tomba 12/1981 dalla necropoli di via Traiana, sempre a Bitonto, con scena erotica a rilievo (cf. RICCARDI 2003, n. 206, fig. 152).

41. Per i valori del kalathos si rimanda anche a BAGGIO 2004, p. 64 ss e p. 142 ss.

42. HALM-TISSERANT 2004.

43. In un ambito culturale assai prossimo, la lucana Paestum, il repertorio delle pitture tombali, in parte confrontabile per orizzonte cronologico con quello della ceramica apula qui considerata, riserva, a partire dalla metà del IV secolo a.C., uno spazio particolare all'iconografia della donna, che viene consacrata nelle immagini come padrona di casa, seduta e intenta a filare la lana accudita da un'ancella, oppure da morta, nel momento della prothesis, circondata da quegli oggetti, che ne definiscono la posizione nell'ambito dell'oikos e della compagine sociale di appartenenza : PONTRANDOLFO, ROUVERET 1992.

44. RVAp II, pl. 330.

45. Qui compare il motivo del "sedersi su vaso", assai frequente nell'immaginario figurato apulo, tuttavia non ancora indagato nel suo simbolismo ; in particolare l'hydria, come è noto, gioca un ruolo molto importante nei rituali di purificazione ed è strettamente connessa alle cerimonie nuziali. Per restare al pittore di Baltimora, sono frequenti le donne sedute su hydriai : entro naiskos (Bari collezione Cavalcanti 14 dans RVAp II, 27/9, pl. 321); offerenti al di fuori del naiskos (Fiesole Collezione Costantini 152 e 153, Mattinata, collezione Sansone 685, Bari collezione Macinagrossa 26 dans RVAp II, 27/54-57, pl. 331, 1-4) ; al di fuori del « Palazzo di Ade » (Malibu 77 AE 13, dans RVAp II, 27/17, pl. 323, e Svizzera, Collezione privata RVAp II, 27/22a, pl. tav 325, 1; in un corteo di giovani e fanciulle (Taranto 9847 dans RVAp II, 27/44, pl.329; donna da sola (Hildesheim RM15 dans RVAp II, 27/116, pl. 337).

46. Si osserva, in corrispondenza delle teste delle due donne, la presenza di due segni grafici, interpretati come finestre. Sul significato delle finestre nella ceramica magnogreca si veda CASSIMATIS 1995, per la quale questo segno non sarebbe un semplice elemento riempitivo, privo di significato, ma un simbolo di tramite, di passaggio e quindi di apertura verso l'al di là.

47. Il sistema di segni raffigurato all'interno del naiskos trova corrispondenza nel sistema di oggetti rinvenuti in alcuni corredi femminili apuli. In generale, sulla presenza di cassettine, cofanetti e contenitori nelle sepolture si veda Marisa CORRENTE (2003). Le cassettine, in particolare, sono spesso documentate dal rinvenimento nelle tombe di serrature in bronzo, dai chiodi, della pannellatura e dai listelli in osso, nonché dal contenuto, costituito da oggetti per la cosmesi e da elementi per il gioco (RICCARDI 2008, p. 32). Una cassetta lignea, uno specchio ed uno strigile costituiscono il sistema di oggetti che caratterizza il corredo femminile della deposizione B/1 dell'Ipogeo dei Serpenti (CORRENTE 2003, p. 35). La deposizione B/2 del medesimo ipogeo presenta una cassetta associata a specchio ed unguentari, elementi funzionali al costume funerario femminile (CORRENTE 2003, p. 35).

48. Si tratta di oggetti simbolo, che richiamano evidentemente i giochi infantili, dedicati da parte di giovani fanciulle al momento della transizione di status, vedi infra nota 52.

49. Secondo un sistema associativo che come già ho dimostrato in altra sede rimanda ad un contesto nuziale: BAGGIO 2004, passim.

50. Lo specchio è certamente l'oggetto più ambiguo: come ha dimostrato Héléne CASSIMATIS (1988), che ha suggerito la possibilità di attribuire a questo oggetto, in particolare nelle rappresentazioni 
a carattere funerario, un significato magico religioso, che ne fa strumento in grado di favorire la comunicazione tra vivi e morti o come riferimento a concezioni escatologiche orfico-dionisiache. 51. Si deve a SCHNEIDER-HERMANN (1971) una prima messa a punto del significato di tale segno nella ceramica apula, da intepretare forse come indice di un momento di passaggio di status: la studiosa ritiene, infatti, che tale oggetto si possa interpretare come il ricordo visivo dell'adolescenza ormai abbandonata, mentre - nelle scene a tema nuziale - possa funzionare con il significato di oracolo amoroso ; senza trascurare un possibile significato funerario come dono di consacrazione definitivo per la defunta. In particolare sulla presenza della palla in raffigurazioni che mettono in scena l'unione amorosa si veda anche BAGGIO 2004 e, da ultimo, PICAUD 2004.

52. Sul possibile significato di questo oggetto, in particolare nelle scene di seduzione erotica si veda BAGGIO 2004. Sul legame cintura/nozze non consumate si veda, tra gli altri, un significativo passo dell'Antologia Palatina (VII, 182) : « Non le nozze conobbe, ma l'Ade marito Clearista come il cinto di vergine disciolse... ».

53. La rappresentazione di una testa femminile tra girali è molto diffusa tra i ceramografi tardo apuli, forse ad esprimere la bellezza della vita oltremondana: sul possibile significato anche AELLEN 1994. Nell'ambito della ceramografia tardo apula il tema appare sul collo dei grandi crateri del pittore di Licurgo, di Baltimora, di Ganimede e della Patera per poi banalizzarsi nella più corsiva produzione apula finale : si veda RVAp I, pp. 189-190 ; RVAp II, p. 447; ROUVERET 1990, p. 317-349; PONTRANDOLFO 1988.

54. La loutrophoros è decorata, nell'altro lato, da una scena di culto alla stele, secondo uno schema utilizzato in età tardo apula negli esemplari più impegnativi: si veda PONTRANDOLFO et alii 1988.

55. Matera, Museo Nazionale « Domenico Ridola », inv. 164531.

56. E' molto difficile definire che rapporto intercorra tra le donne: sono compagne, parenti, serve?

57. Ricordiamo che tra i doni fatali offerti da Medea a Glauce, in occasione dello sfortunato matrimonio con Giasone, figura una corona d'oro preziosamente cesellata: Euripide, Medea, 947-958.

58. Coppie di orecchini in oro e pietre preziose provengono, ad esempio, da alcuni contesti sepolcrali rinvenuti nel sito di Ruvo: si veda CASSANO 1996, p. 123.

59. I corredi funebri femminili della zona canosina hanno restituito preziose applicazioni di tessuto in oro e fibre tessili, sempre in oro, che indicano il rango elevato delle deposizioni in contesti emergenti: si vedano LIPPOLIS 1994 p.339, n. 277 e CORRENTE 2003, p. 67. Sul ruolo delle vesti quali indicatori d'identità e statuto in Grecia e a Roma si vedano anche GHERCHANOC, HUET 2009.

60. La tomba 7/2003 rinvenuta a Bitonto ed attribuita ad una sepoltura femminile conteneva, oltre ad un anello in bronzo, un vago in pasta vitrea di produzione orientale, certamente con l'intento di distinguere in maniera inequivocabile il rango della defunta (RICCARDI 2008).

61. Come potrebbe indicare il rinvenimento di piccoli kalathoi analoghi, in terracotta, rinvenuti nei santuari magno greci, in connessione con il culto di Kore. D'altra parte, la presenza del kalathos nelle immagini relative al ratto di Persefone da parte di Ade, richiama tanto la simbologia nuziale, quanto quella funeraria considerando che la dea è sposa di Ade, ma anche signora degli Inferi. Al tema della fecondità sembrerebbe rimandare anche la presenza di kalathoi ricolmi di frutti nelle scene d'incontro tra i sessi, assai frequenti nella ceramica apula : si veda, tra gli altri, CIANCIO 1997, p. 89, fig. 113.

62. La frequente comparsa nell'iconografia apula di strumenti musicali, come la lira o la cetra, sembrerebbe documentare una maggiore importanza attribuita alla musica nella società aristocratica magnogreca nei decenni finali del IV secolo a.C., come suggerisce Gemma SENA CHIESA (2006, p. 393). Tuttavia, poiché la cetra compare come strumento nelle mani di Orfeo (ad es. 
nel cratere a volute apulo attribuito al Pittore di Licurgo datato al 360-350 a.C. dans SENA CHIESA 2006, p. 316 s.), non possiamo escludere per questo oggetto una simbologia misterica di stampo orfico, con riferimento ad un significato funerario ed escatologico.

63. La moltiplicazione degli unguentari insieme alla deposizione del cofanetto ligneo sembrerebbero rispondere nei corredi tombali a nuove esigenze rappresentative della cultura daunia in età ellenistica : CORRENTE 2003, p. 125 ss.

64. Vedi supra, nota 26.

65. Per qualche esempio tratto da Euripide si vedano: Alcesti 149, 613 ; Ecuba 578, 615 ; Eracle 329, 334,548 , Supplici 78.

66. RVAp II, p. 726, n. 23/6. Per il contesto di ritrovamento, una tomba a semicamera di grandi dimensioni rinvenuta a Ruvo nei pressi di Via Gravinelle, già violata in antico, si veda il recente volume di A. MONTANARO (2007, in particolare p. 406-407 e p. 409-410).

67. RVAP II, p. 721.

68. Per quanto siano ancora pochi i luoghi certi di provenienza dei vasi del pittore della Patera, più dei due terzi dei vasi sul totale noto vengono da Ruvo.

69. SCHNEIDER-HERMANN 1961 ; eadem 1975.

70. Si veda RVAp II, tav. 331, 1-4

71. Il tema non è molto diffuso all'interno della produzione del pittore di Baltimora: si ricorda un cratere a volute apulo, ora Collezione Banca Intesa 109, dove il tema delle fanciulle alla fonte è associato ad una scena di amazzonomachia, e due crateri di San Pietroburgo B 1716 e 1717.

72. Si vedano, da ultimo, i contributi raccolti nel volume Cultura e religione delle acque 2012. Si rimanda inoltre a MENICHETTI 2006, con bibliografia precedente.

73. Il mito di Niobe gode di grande fortuna nella tradizione letteraria: Eschilo e Sofocle scrissero una Niobe, di cui oggi rimangono scarsissimi frammenti : Eschl., TrGF III, F 154a- 167 b ; Sof., TrGF IV, F 441a- 451. Dall'Antigone, vv. 823-833 : ho udito che molto compianta perì l'ospite frigia la figlia di Tantalo, sulla vetta del Sipilo; com'edera avvolgendola la domò la vegetazione di pietra; corrosa, mai la pioggia e la neve la abbandonano, ma dalle ciglia un pianto continuo bagna il suo collo di roccia; dall' Elettra, vv. 150-152: O Niobe infelice, che nel tuo sepolcro di roccia, ahimè piangi una dea ti considero ; Euripide nelle Fenice 159-161 la ricorda vicino alla tomba delle sette fanciulle di Niobe. Scriveranno di Niobe: Ferecide (FGrHist, 3 F 38), Ellanico (FGrHist, 4 F21); Timoteo di Mileto, autore di un

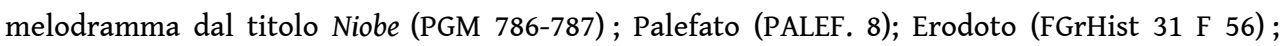
Diodoro Siculo (IV, 74) ; Lattanzio Placido (Latt. Comm. Ad Theb. III, 191) ; Giovanni Tzetze (Chil. IV, 416).

74. Sul mito di Niobe in Magna Grecia si vedano : AELLEN 1994; MAZZEI 1999 ; GUALTIERI 2003 con bibliografia precedente; POUZADOUX 2005 ; MORARD 2009. In generale sul mito in Apulia scrive Marina MAZZEI (1999, p. 470) : «...ad una presenza del mito attestata già dall'inizio della produzione,...segue un incremento che si consolida col pittore di Dario, si calibra col suo più importante successore, il pittore di Baltimora, e si reduce confermando alcuni temi specifici col pittore di Arpi... ». In relazione al pittore di Baltimora la studiosa scrive (p. 470) : « ...pare operare una selezione tra i tanti temi e sembra consolidare nettamente soprattutto quelli già adottati dal pittore di Dario. Continua la tradizione troiana e la tradizione eraclea, con lui si afferma il mito delle Danaidi e si adotta, per la prima volta dopo il pittore Varrese, il mito di Niobe... ».

75. La più antica raffigurazione del mito si ha su una neck-amphora rinvenuta in un contesto tombale a Roccagloriosa, nella Lucania occidentale, che ritrae Niobe nel momento della pietrificazione, raffigurata su un'alta base. A sinistra dell'eroina sono una figura femminile, da interpretarsi come la madre, la matrigna o la nutrice dei figli ; a destra, inginocchiato in gesto di supplica è un vecchio con barba bianca da interpretarsi come Tantalo, il padre di Niobe. Su questo documento si vedano da ultimo GUALTIERI 2003 e GUALTIERI 2006. 
76. C'è un evidente scarto tematico rispetto ai vasi di produzione attica di v secolo a.C., dove i pittori avevano privilegiato il momento della strage dei figli di Niobe (come si osserva, ad esempio, nel famosissimo cratere a calice attribuito al pittore dei Niobidi, rinvenuto ad Orvieto ed ora conservato a Parigi, Museo del Louvre (per cui si veda, da ultimo, DENOYELLE 1997), oppure il frammento di un cratere attribuito al Pittore di Bologna 276, ora a Rimini, Museo Missionario alle Grazie 07088, con Apollo e Niobide caduto (per cui si veda: ARV ${ }^{2}$ 611.40bis ). Il tema doveva decorare anche parte del trono di Zeus opera di Fidia, come racconta Pausania $(V, 11,2): s u$ ognuno dei due piedi anteriori dei fanciulli tebani rapiti da sfingi e sotto le sfingi Apollo e Artemide che uccidono a colpi di freccia i figli di Niobe. Meno ricca la documentazione di ambito apulo, dove la strage dei Niobidi compare in un cratere a volute attribuito al pittore di Baltimora (Ruvo, Collezione Jatta J424, inv. 36735), per il quale si veda da ultimo MORARD 2009, p. 92, cat. 66, tav. 50 ; per la raffigurazione su un'hydria del pittore di Arpi, si veda TODIsCo 2008.

77. Frammento di anfora a collo distinto da Roccagloriosa (sснміDт 1992, n. 15, p. 911) ; anfora del pittore di Varrese (355-345 a.C.), Bonn, Akademisches Kunstmuseum (scHMIDT 1992, n. 16, p. 911); loutrophoros del pittore del Louvre MNB1148, Malibu J. Paul Getty Museum (schmidT 1992, n. 18, p. 912); hydria del pittore di Dario (340 a.C.), Ginevra collezione privata (schmidT 1992, n. 13, p. 911 ; Le peintre de Darius 1986, p. 150 ss.); hydria del pittore di Ganimede (340-320 a.C.) Zurigo, Archäologische Sammlung der Universität (SCHMIDT 1992, n. 19); hydria campana del pittore della Libagione, Sydnei Nicholson Museum (sснміDт 1992, n. 11, p. 911); lekythos del pittore di Caivano, Berlino Staatliche Museum (schmidT 1992, n. 17).

78. Del vaso fortunatamente è noto il contesto di provenienza: si tratta di una neck-amphora a figure rosse rinvenuta nella monumentale tomba a camera 24 delle necropoli di Roccagloriosa, una sepoltura di alto livello pertinente ad una figura femminile. Sul contesto di rinvenimento si veda in particolare GUALTIERI 2003, p. 147-153, con bibliografia precedente e GUALTIERI 2006. Sull'immagine di Niobe in questo vaso si veda anche DE CESARE 1997, p. 154, fig. 54.

79. Attribuito al pittore della Libagione, per cui vedi infra.

80. MAZZEI 1999 ; MORARD 2009.

81. Napoli, Museo Archeologico Nazionale 82267 (H 3246): schmidt 1992, p.911, n. 12 ; MONTANARO 2007, p. 296 s. per il contesto di rinvenimento. Alla bottega del pittore di Varrese si attribuiscono altri due vasi che mettono in scena il mito di Niobe: un'anfora apula, proveniente da Canosa, ora conservata ora a Taranto, Museo Archeologico Nazionale inv. 8935 (per cui si veda SCHMIDT 1992, p. 910, n. 10 e DE CESARE 1997, p. 156, fig. 95), dove Niobe non è raffigurata stante bensì seduta su un altare, collocato sopra un monumento sepolcrale sostenuto da colonne e da una trabeazione di triglifi e metope. Qui la figlia di Tantalo è raffigurata tra due anfore, nel gesto codificato dell'afflizione. In questo caso, come sottolinea Monica DE CESARE (1997, p. 156), la resa figurativa non consente di stabilire se sia raffigurata una statua o un'immagine ancora vivente. Degno di nota il fatto che una serie di oggetti appoggiati a terra o sospesi punteggino la raffigurazione: una patera, un kalathos di grandi dimensioni raffigurato tra un vecchio con barba bianca, forse Tantalo, e Niobe ; sotto il monumento, nello spazio tra le colonne, si riconoscono uno specchio, una cassettina aperta, un drappo ed un'hydria. In un'altra loutrophoros ancora attribuita al pittore di Varrese, Niobe è raffigurata entro un naiskos tra due loutrophoroi (Bonn 99, in KEULS 1989).

82. Degno di nota l'alto basamento su cui poggia il naiskos, decorato su almeno i due lati visibili da un motivo di sfingi che emergono da girali vegetali. Secondo MORET 1984, p.99, proprio nell'ambito apulo la sfinge sembra assumere una decisa connotazione escatologica e viene frequentemente usata come ornamento dell'edificio funerario: come acroterio del naiskos, in una hydria attribuita al pittore di Varrese (Taranto 8922, RVAp I, tav. 108,1) ; collocata all'interno del naiskos, in un cratere a volute ora a New York Coll. R. Curie (RVAp, Suppl I, 30/98 b) ; come ornamento delle colonne del Palazzo di Ade in un cratere a calice frammentario attribuito al 
pittore della Nascita di Dioniso (Taranto 54.959, RVAp I, 2/26), su un cratere a volute Karlsruhe B4 attribuito alla cerchia del pittore di Licurgo (RVAp I, pl. 160, 1), su un frammento di vaso attribuito al pittore di Dario (New York L.1972.30.2, RVAp II, tav.181, 2), dove le sfingi compaiono, con evidente significato simbolico, come ornamento al trono di Persefone entro il palazzo di Ade.

83. Sulla metamorfosi di Niobe si veda anche FRONTISI-DUCROUX 2003. Si dibatte se si tratti del monumento sepolcrale dell'eroina (così KEULS 1978 e (Le) peintre de Darius et son milieu 1986) o dei Niobidi (così FRONTISI-DUCROUX 2003, p. 145).

84. Sul ruolo delle assemblee divine nel mito di Niobe si veda da ultimo: MORARD 2009, p. 80 s., 92 s. e passim.

85. Già SCHMIDT 1992, p. 914.

86. Lo scettro che il vecchio stringe con la mano, in questa immagine come in alcune altre (cf. almeno sCHміDт 1992, n. 11, 12, 13, 17), potrebbe evocare visivamente la discendenza divina.

87. Per cui anche POUZADOUX 2005.

88. In generale, la lira è un oggetto assai frequente nelle raffigurazioni apule: come strumento nelle mani di Apollo (in un cratere a volute del pittore di Palermo in TRENDALL 1989, fig. 20; in un cratere a calice frammentario attribuito al pittore della Nascita di Dioniso in RVAp I, tav 9, 2 TRENDALL 1989, fig. 52 ; TRENDALL 1989, fig. 210 ; in un cratere a volute del pittore di Capodimonte, in TRENDALL 1989, fig. 260). E' strumento nelle mani di Orfeo (in un cratere a volute frammentario del Gruppo di Locri, TRENDALL 1989, fig. 63 ; suonatore di lira in un naiskos nell'hydria del pittore del Primato, TRENDALL 1989, fig. 98 ; tenuta in mano nella oinochoe del pittore Felton, TRENDALL 1989, fig. 136 ; appoggiata al suolo in una hydria del pittore di Varrese (TRENDALL 1989, fig. 172 ; associata al mito di Niobe in una loutrophoros del Pittore del Louvre MNB 1148 (TRENDALL 1989, fig. 183 dove osserviamo sia il raddoppiamento della lira sia la lira associata al kalathos; in basso tema nuziale; entro naiskos sospesa nel campo con giovane presso il louterion e cane in un cratere a volute del Pittore di Copenhagen 4223, TRENDALl 1989, fig. 189).

89. Nelle immagini prodotte in ambito apulo la corazza è associata a giovani uomini raffigurati entro naiskoi : si vedano in proposito: un'anfora attribuita al pittore H.A. (TRENDALL 1989, fig. 164); un cratere a volute del pittore di Baltimora (TRENDALL 1989, fig. 250); un cratere a volute del pittore di Dario decorato con i funerali di Patroclo (TRENDALl 1989, fig. 204, dove figura come elemento di un'intera panoplia). Spesso è raffigurata sospesa entro naiskos come nel caso di un cratere a volute del pittore di Ganimede (TRENDALL 1989, fig. 238 e 239 ; TRENDALL 1989, fig. 256) ; talvolta anche da sola entro naiskos (per cui RVAp II, tav. 214, 1-2).

90. Sugli oggetti significanti nell'immagine si veda LISSARRAGUE 2006.

91. Senza trascurare il fatto che la lira è frequente strumento nelle mani di Orfeo e dunque la sua presenza in immagini col mito di Niobe potrebbe ricollegarsi a forme di religiosità orficosalvifiche, cui non dovevano essere estranei i ceti più elevati della committenza apula: si veda in proposito MUGIONE 2000, con bibliografia di riferimento.

92. Sulla corazza utili considerazioni in CASSANO 1992, p. 261.

93. La corazza anatomica, come è noto, è destinata alle figure dei cavalieri nelle comunità apule. Come ha ben evidenziato la recente letteratura critica (GUZzo 1993), per quanto il numero delle corazze note sia estremamente ridotto, si osserva di contro una concentrazione eccezionale di queste nelle tombe canosine, dato che potrebbe far ipotizzare la localizzazione delle officine di produzione nella stessa Canusium (per cui si veda 1912 Un ipogeo al confine 2000).

94. Sulla presenza delle armi, tanto nei corredi tombali quanto nel sistema delle immagini delle comunità indigene della Basilicata, come simboli dell'autorappresentazione maschile si veda anche COLIVICCHI 2009.

95. Oggetto di dibattito è l'identificazione della tomba su/in cui è raffigurata Niobe : Eva KEULS (1978) e Christian AELLEN (1994) ritengono si tratti della tomba di Niobe stessa, in quanto l'eroina 
qui sarebbe rappresentata come defunta. TRENDALL (1985) e FRACCHIA (1989) ritengono si tratti della tomba dei figli. Ritengo che la presenza di oggetti maschili, come ad esempio la corazza dipinta ai piedi del naiskos nella loutrophoros attribuita al pittore di Varrese vedi supra, potrebbe far propendere per questa seconda ipotesi.

96. Zurich, Archäologische Sammlung der Universität 4007: SCHMIDT 1992, p. 912, n. 19 (particolare).

97. Per i valori semantici del bianco vedi supra nota 30. Sulla possibilità di interpretare la fanciulla come una Tantalide si veda SCHMIDT 1992, p. 911, n. 11.

98. Sydney, The Nicholson Museum, inv. n. 71.01.

99. Sul rapporto tra produzione attica e produzione magnogreca in relazione alla distribuzione degli oggetti mi riservo di intervenire in altra sede. Sulle lekythoi attiche si veda da ultimo: OAKLEY 2004.

100. Scrive in proposito Marina MAZzEI (1990, p. 155): «la committenza agisce nella piena consapevolezza nell'adozione delle forme vascolari e delle immagini, che pertanto non sono isolate attestazioni di un mondo provinciale, ma l'espressione dei centri egemoni della Puglia settentrionale... ».

\section{RIASSUNTI}

Le système figuratif d'un groupe de vases apuliens daté de la seconde moitié du IV siècle av. J.-C., en particulier les vases de l'atelier du peintre de Baltimore, qui donnent un rôle clé à la disposition des objets dans l'image, forme un observatoire privilégié pour jeter un nouvel éclairage sur les mécanismes de représentation des femmes.

The figurative system of Apulian vases dating back to the second half of the fourth century B.C., specifically the Baltimore Painter's vases, constitutes a privileged observatory to shed new light on the mechanisms of feminine representation.

\section{INDICE}

Keywords : south Italian vase, Apulia, loutrophoroi, iconography, gender, object

Mots-clés : grande Grèce, Apulie, céramique, iconographie, loutrophore, peintre de Baltimore, femme, objet

\section{AUTORE}

MONICA BAGGIO

ANHIMA - UMR 8210 